\title{
Ectopic Expression Induces Abnormal Somatodendritic Distribution of Tau in the Mouse Brain
}

\author{
Atsuko Kubo, ${ }^{1}$ Shouyou Ueda, ${ }^{1}$ Ayaka Yamane, ${ }^{2}$ Satoko Wada-Kakuda, ${ }^{1}$ Mai Narita, ${ }^{1}$ Makoto Matsuyama, ${ }^{3}$ \\ Akane Nomori, ${ }^{3}$ Akihiko Takashima ${ }^{4,5}$ Taisuke Kato, ${ }^{6}$ Osamu Onodera, ${ }^{6}$ Motohito Goto, ${ }^{7}$ Mamoru Ito, ${ }^{7}$ \\ Takami Tomiyama, ${ }^{8}$ Hiroshi Mori, ${ }^{8}$ Shigeo Murayama, ${ }^{9}$ Yasuo Ihara, ${ }^{1}{ }^{\circ}$ Hiroaki Misonou, ${ }^{2,5}$ \\ and $\odot$ Tomohiro Miyasaka ${ }^{1,5}$ \\ ${ }^{1}$ Department of Neuropathology, Faculty of Life and Medical Sciences, Doshisha University, Kyotanabe-shi, Kyoto 610-0394, Japan, ${ }^{2}$ Laboratory of Ion \\ Channel Pathophysiology, Graduate School of Brain Science, Doshisha University, Kyotanabe-shi, Kyoto 610-0394, Japan, ${ }^{3}$ Division of Molecular Genetics, \\ Shigei Medical Research Institute, Minami-Ku, Okayama 701-0202, Japan, ${ }^{4}$ Department of Alzheimer's Disease, Faculty of Life Science, Gakushuin \\ University, Toshima-ku, Tokyo 171-0031, Japan, ${ }^{5}$ Center for Research in Neurodegenerative Diseases, Doshisha University, Kyotanabeshi, Kyoto 610-0394, \\ Japan, ${ }^{6}$ Department of Neurology, Clinical Neuroscience Branch, Brain Research Institute, Niigata University, Niigata 951-8585, Japan, ${ }^{7}$ Central Institute for \\ Experimental Animals (CIEA), Tonomachi, Kawasaki-ku, Kawasaki, 210-0821, Japan, ${ }^{8}$ Department of Neuroscience, Graduate School of Medicine, Osaka \\ City University, Abeno-Ku, Osaka 545-8585, Japan, and ${ }^{9}$ Neuropathology, The Brain Bank for Aging Research, Tokyo Metropolitan Geriatric Hospital, \\ Tokyo 173-0015, Japan
}

Tau is a microtubule (MT)-associated protein that is localized to the axon. In Alzheimer's disease, the distribution of tau undergoes a remarkable alteration, leading to the formation of tau inclusions in the somatodendritic compartment. To investigate how this mislocalization occurs, we recently developed immunohistochemical tools that can separately detect endogenous mouse and exogenous human tau with high sensitivity, which allows us to visualize not only the pathological but also the pre-aggregated tau in mouse brain tissues of both sexes. Using these antibodies, we found that in tau-transgenic mouse brains, exogenous human tau was abundant in dendrites and somata even in the presymptomatic period, whereas the axonal localization of endogenous mouse tau was unaffected. In stark contrast, exogenous tau was properly localized to the axon in human tau knock-in mice. We tracked this difference to the temporal expression patterns of tau. Endogenous mouse tau and exogenous human tau in human tau knock-in mice exhibited high expression levels during the neonatal period and strong suppression into the adulthood. However, human tau in transgenic mice was expressed continuously and at high levels in adult animals. These results indicated the uncontrolled expression of exogenous tau beyond the developmental period as a cause of mislocalization in the transgenic mice. Superresolution microscopic and biochemical analyses also indicated that the interaction between MTs and exogenous tau was impaired only in the tau-transgenic mice, but not in knock-in mice. Thus, the ectopic expression of tau may be critical for its somatodendritic mislocalization, a key step of the tauopathy.

Key words: Alzheimer; axon; tau; tauopathy

\section{Significance Statement}

Somatodendritic localization of tau may be an early step leading to the neuronal degeneration in tauopathies. However, the mechanisms of the normal axonal distribution of tau and the mislocalization of pathological tau remain obscure. Our immunohistochemical and biochemical analyses demonstrated that the endogenous mouse tau is transiently expressed in neonatal brains, that exogenous human tau expressed corresponding to such tau expression profile can distribute into the axon, and that the constitutive expression of tau into adulthood (e.g., human tau in transgenic mice) results in abnormal somatodendritic localization. Thus, the expression profile of tau is tightly associated with the localization of tau, and the ectopic expression of tau in matured neurons may be involved in the pathogenesis of tauopathy. 


\section{Introduction}

Tau is a microtubule (MT)-associated protein that is preferentially expressed in brain cells; within neurons, tau is exclusively expressed in the axons (Abraha et al., 2000; Zilka et al., 2006; Cohen et al., 2011; Scholz and Mandelkow, 2014). Tau is also known as a component of the paired helical filament that is found in neurofibrillary tangles (NFTs) or neuropil threads in "tauopathies," including Alzheimer's disease (AD) (Johnson and Jenkins, 1999). Both pathological evidence, which indicates a strong correlation between the formation of tau pathologies and neuronal degeneration (Gómez-Isla et al., 1997; Delacourte et al., 1999), and genetic evidence from familial dementia strongly suggest that some sort of tau abnormalities can directly cause neurodegeneration and dementia (Ghetti et al., 2015).

Despite the axonal localization of tau in normal neurons, in $\mathrm{AD}$ and other tauopathies, tau inclusions are formed in the somatodendritic compartments of affected neurons (Kowall and Kosik, 1987; Braak and Braak, 1994; Attems, 2013). Cumulative evidence indicates that the formation of NFTs itself might not directly cause neuronal dysfunction and degeneration (Miyasaka et al., 2005a; Santacruz et al., 2005; Kuchibhotla et al., 2014) and that the abnormal distribution of presumably unaggregated tau into dendrites or spines is a critical determinant for neurodegeneration (Zempel et al., 2010; Frandemiche et al., 2014). Therefore, the "mislocalization" of tau may be a key step in the pathogenesis of tauopathies (Zempel and Mandelkow, 2014).

To determine how axonal tau mislocalizes to and accumulates in the somatodendritic compartment, we analyzed the distribution of endogenous tau and compared it with that of exogenously expressed human tau in widely used mouse models of tauopathy. Although several previous studies have shown the overall distribution of tau in normal brain tissues (Binder et al., 1985; Kowall and Kosik, 1987; Viereck et al., 1988; Trojanowski et al., 1989), the precise subcellular localization of endogenous tau and how this localization pattern changes in tauopathy models have not yet been extensively demonstrated, presumably due to the poor antigenicity of unaggregated endogenous tau (Trojanowski et al., 1989). We have recently developed new antibodies against tau and optimized procedures to reliably detect endogenous normal, unaggregated tau and expressed human tau separately in brain tissues (Kubo et al., 2019). Here, using these new tools, we investigated how the localization of tau is affected in the animal models of tauopathy.

\section{Materials and Methods}

Animals and human tissues. The following animals were used in this study: P301L tau-transgenic mice (P301L-Tg; Kimura et al., 2010), wildtype tau-transgenic mice (WT-Tg; Kimura et al., 2007), tau knock-out

Received Nov. 4, 2018; revised June 13, 2019; accepted June 18, 2019.

Author contributions: A.K., S.U., A.Y., S.W.-K., M.N., H. Misonou, and T.M. performed research; A.K., S.U., A.Y., S.W.-K., H. Misonou, and T.M. analyzed data; A.K. wrote the first draft of the paper; M.M., A.N., A.T., T.K., 0.0., M.G., M.I., T.T., H. Mori, and S.M. contributed unpublished reagents/analytic tools; Y.I., H. Misonou, and T.M. designed research; H. Misonou and T.M. wrote the paper.

This work was supported in part by a Grant-in-Aid for Scientific Research on Innovative Areas "Brain Protein Aging and Dementia Control" (Grant 26117004 to T.M.), "Integrated Research on Neuropsychiatric Disorder," which was performed under the Strategic Research Program for Brain Sciences (T.M. and Y.I.), JSPS Core-to-Core Program A Advanced Research Networks, the Strategic Research Foundation at Private Universities (S1201009), the Mitsubishi Foundation (T.M.), and the Japan Society for the Promotion of Science (KAKENHI Grant 22650074 to T.M., Grant 26640030 to A.K., and Grant 16 K07006 to H.M.). We thank Akihiro Harada and Fumitaka Oyama for tau-KI mice and Tsuyoshi Waku, Shinske Ishigaki, and Gen Sobue for technical assistance.

The authors declare no competing financial interests.

Correspondence should be addressed to Tomohiro Miyasaka at tomiyasa@mail.doshisha.ac.jp.

https://doi.org/10.1523/JNEUROSCI.2845-18.2019

Copyright $(2019$ the authors mice (tau-KO; Dawson et al., 2001), line 264-Tg tau mice (Tau264; Umeda et al., 2013), and PS19 mice. PS19 mice, which express P301S tau under the mouse prion promoter, were from The Jackson Laboratory (Yoshiyama et al., 2007). Tau knock-in mice (P301L-KI and V337M-KI) were developed by inserting human CDNA and a floxed PGK-Neo poly-A cassette in the same region as the previous tau-KO mice (Harada et al., 1994). Knock-in mice were developed by in-frame insertion of human cDNA for 0N4R isoform (383 aa) followed by rabbit $\beta$-globin poly-A signal sequence from the mouse endogenous start codon in the first coding exon. Floxed PKG-Neo poly-A was inserted after the human tau cDNA (Matsumura et al., 1999). We also generated crossbred of heterozygous P301L-KI mice and APP-Tg mice (Tg-2576; Hsiao et al., 1996). All animal experiments were approved by the institutional animal care and use committees. Both male and female animals were used. The autopsy brain tissues were obtained from the Brain Bank for Aging Research, Tokyo Metropolitan Institute of Gerontology (TMIG), Japan (www.mci.gr.jp/BrainBank/index.cgi) with written informed consents for their use in medical research from the patients or their families. Their use in this particular study was approved by the ethics committee at Doshisha University and TMIG.

Antibodies. Anti-tauN and anti-MAP2N rabbit polyclonal antibodies were raised against the $\mathrm{N}$-terminal peptide (AEPRQEFEVMEDHAGGGC) of human tau and the N-terminal 150 aa fragment of recombinant human MAP2, respectively. Anti-rodent tauN (anti-RtauN) rabbit polyclonal antibody was raised against the peptide (DTMEDHAGDYTLLQDEG) corresponding to the N-terminal potion of mouse tau. Anti-total tau (RTM38), anti-human tau specific (RTM49), and anti-mouse tau specific (RTM47) rat monoclonal antibodies were raised against purified recombinant human and mouse tau, respectively. We have reported the production and validation of these antibodies recently (Kubo et al., 2019). The anti-tau rat IgGs are now commercially available from FUJIFILM Wako Pure Chemical Corporation.

Antibody dilutions. Dilutions of the antibodies for immunostaining are listed below; anti-tauN, anti-MAP2N, and anti-RtauN (1:1000), antitau12 (tau12, mouse monoclonal antibody, 1:2000, Abcam), tau1 (tau1, mouse monoclonal antibody, 1:1000, Millipore), anti-MAP2 (HM2, mouse monoclonal antibody, 1:1000, Sigma-Aldrich), RTM49, RTM47 and RTM38 (1:300), tau5 (tau5, mouse monoclonal antibody, 1:1000, Millipore), BD-anti-tau (anti-tau, mouse monoclonal antibody, 1:1000, BD Biosciences).

Preparation of tissue sections and neuronal primary cultures. Animals were anesthetized with pentobarbital and fixed via transcardial perfusion with 4\% paraformaldehyde (PFA) in PBS. Heads were postfixed in the same fixative for $48 \mathrm{~h}$ at room temperature. Brains were harvested and sliced into 50 - $\mu \mathrm{m}$-thick sections using a vibrating microtome (LinearSlicer Pro7, Dosaka), and sections were stored in PBS. Some sections were dehydrated and embedded in paraffin.

Dissociated cultures of embryonic ( E17-E18) mouse hippocampal neurons were prepared from female timed pregnant mice of either non-Tg or P301L-Tg as previously described for rat cultures (Misonou and Trimmer, 2005) with minor modifications. Briefly, dissected hippocampi were digested in $0.25 \%$ trypsin for $15 \mathrm{~min}$ at $37^{\circ} \mathrm{C}$, dissociated by pipetting, and then plated onto glass coverslips coated with $1 \mathrm{mg} / \mathrm{ml}$ poly-L-lysine at 50,000 cells/coverslip. Neurons were cultured for 3 to $14 \mathrm{~d}$ in vitro (DIV) in 6-well plates, on the bottom of which contained astrocyte cultures. Coverslips were lifted with wax pedestals as described by Kaech and Banker (2006). Cytosine arabinoflanoside was added to the culture at 2 DIV to prevent the growth of non-neuronal cells on the coverslips. For the expression of GFP-tagged tau, neurons were transfected with a DNA plasmid coding GFP-tagged mouse tau (the 0N4R isoform) using Lipofectamine 2000 (Invitrogen) at 7 DIV. Neurons were fixed with 4\% PFA for immunofluorescence staining.

Immunostaining and visualization of tissue sections and primary cell cultures. The tissue sections and cultured neurons were permeabilized with methanol and blocked in $10 \%$ goat serum in PBS with $0.1 \%$ Tween 20 (PBS-T) for $60 \mathrm{~min}$. They were then incubated with primary antibodies diluted in 1\% BSA-PBS-T for 48 to $72 \mathrm{~h}$ at room temperature. After the sections were rinsed with PBS-T, bound antibodies were visualized with secondary antibodies that were conjugated to Alexa Fluor dyes 


\section{Table 1. Details of primers used for qRT-PCR}

\begin{tabular}{|c|c|}
\hline Target of mRNA & Sequence $\left(5^{\prime}-3^{\prime}\right) /$ orientation \\
\hline Gapdh & $\begin{array}{l}\text { GAAACCTGCCAAGTATGATGACAT/sense } \\
\text { GGAAGAGTGGGAGTTGCTGTTG/antisense }\end{array}$ \\
\hline Camk2a & $\begin{array}{l}\text { CAGCTGATCGAAGCCATAAGC/sense } \\
\text { CAGGGTCGCACATCTTCGT/antisense }\end{array}$ \\
\hline$N t f 3$ & $\begin{array}{l}\text { GTGACAGCACCCCTTTGGA/sense } \\
\text { CGGGTTGCCCACATAATCC/antisense }\end{array}$ \\
\hline Tuba1 & $\begin{array}{l}\text { GGACATGGCTGCCCTAGAGA/sense } \\
\text { CCCTCGCCTTCCACAGAA/antisense }\end{array}$ \\
\hline$T u b b 1$ & $\begin{array}{l}\text { TCACGCAGCAGATGTTCGAT/sense } \\
\text { GTGGCGCGGGTCACA/antisense }\end{array}$ \\
\hline Map1b & $\begin{array}{l}\text { CAACAGTGGACTGGCACCAA/sense } \\
\text { GAAATCGGGATGTCTACTTCTTGAC/antisense }\end{array}$ \\
\hline Map2 & $\begin{array}{l}\text { CCAGGTGGTGGACGTGTGA/sense } \\
\text { CTTGGGCCTTTTCCTTGAAAT/antisense }\end{array}$ \\
\hline Mapt & $\begin{array}{l}\text { GGCACCTCAGCAATGTGTCTT/sense } \\
\text { TGTGGCAAGCTGTGGTGAGT/antisense }\end{array}$ \\
\hline human tau & $\begin{array}{l}\text { CGGAGGAAATAAAAAGATTGAAACC/sense } \\
\text { GTCTTGGCTTTGGCGTTCTC/antisense }\end{array}$ \\
\hline
\end{tabular}

(Thermo Fisher Scientific). The specimens were observed under a BZX800 (Keyence), a LSM700 (Carl Zeiss), a TCS SP8 LASER scanning confocal (Leica Microsystems), or a TCS SP8 stimulated emission depletion (STED) (Leica Microsystems) microscope using $10 \times$ and $40 \times$ dry objectives and $63 \times$ and $100 \times$ oil objectives.

The deparaffinized and hydrated paraffin embedded sections were blocked by $10 \%$ goat serum in Tris-buffered saline (TBS; $150 \mathrm{~mm} \mathrm{NaCl}$, 50 mм Tris, pH7.6). The sections were incubated with primary antibodies diluted in $1 \%$ BSA in TBS with $0.1 \%$ Tween 20 (TBS-T) overnight at room temperature. After brief washing in TBS-T, the sections were immersed in 1\% BSA in TBS-T containing biotinylated second antibodies (Vector Laboratories). Then, the sections were labeled by avidin and biotinylated HRP (Vectastain ABC kit; Vector Laboratories) and developed with 3,3-diaminobenzidine (DAB) in the presence of hydrogen peroxide (Miyasaka et al., 2005b).

MT-binding assay. MT-bound and -unbound tau were prepared as described previously with minor modifications (Miyasaka et al., 2010). Briefly, mouse brains were homogenized in MT stabilization buffer (1 mм $\mathrm{MgSO}_{4}, 1$ mм EGTA, 0.1 mм DTT, 0.5\% Triton X-100, 10\% glycerol, $20 \mu \mathrm{m}$ Taxol, $2 \mathrm{~mm}$ GTP, protease/phosphatase inhibitors, and $0.1 \mathrm{~m}$ MES, $\mathrm{pH}$ 6.8). After a brief centrifugation, the supernatants were then centrifuged at $135,000 \times g$ for $15 \mathrm{~min}$ at $2^{\circ} \mathrm{C}$. The levels of tau in the supernatant (MT-unbound fraction) and in the pellet (MT-bound fraction) were quantified by Western blotting with purified recombinant tau as standards.
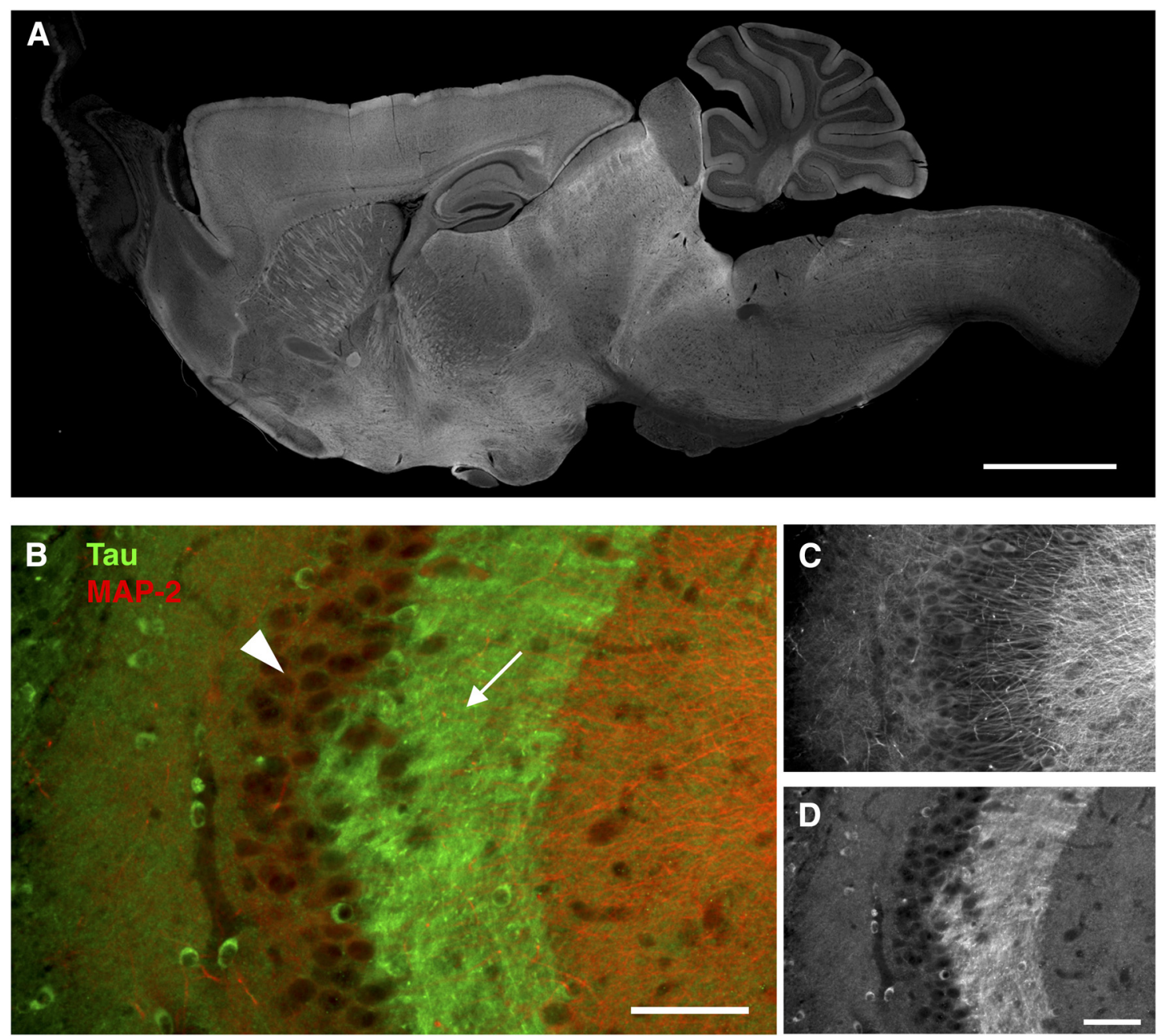

Figure 1. Axonal localization of endogenous tau revealed by our antibodies. $A$, The overall tissue distribution of endogenous tau in the mouse brain. Sagittal brain sections from perfusion-fixed mice were immunostained with RTM38 anti-tau antibody. Scale bar, $1 \mathrm{~mm}$. $\boldsymbol{B}-\boldsymbol{D}$, Distribution of endogenous tau in the hippocampal CA3. Tau immunoreactivity ( $g r e e n$ and $\boldsymbol{D}$ ) was abundant in the mossy fiber axons (arrow) but not in the pyramidal layer (arrowhead), where MAP-2 labeling (red and $C$ ) is prominent. Scale bars, $100 \mu \mathrm{m}$. 

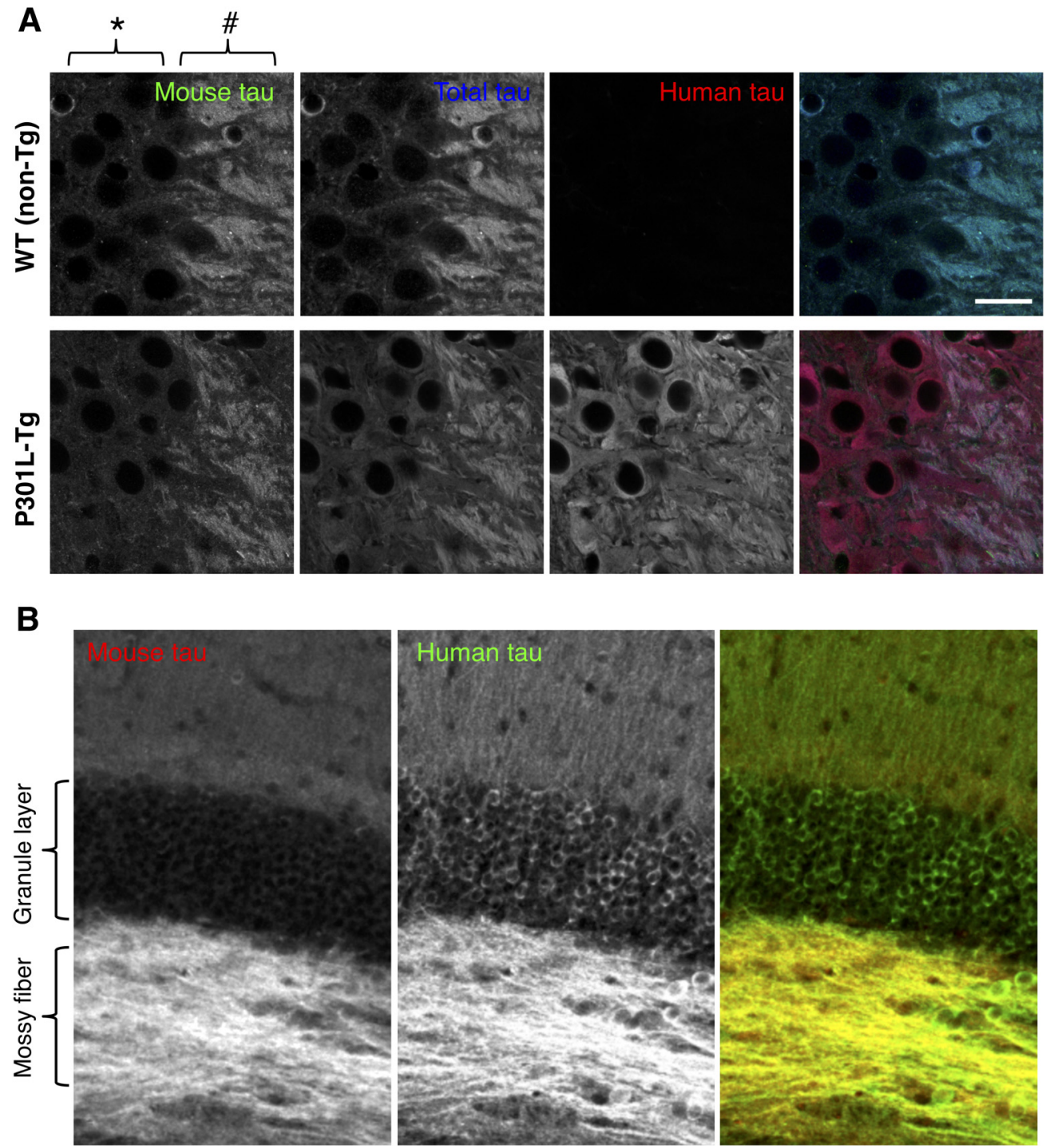

Figure 2. Abnormal somatodendritic localization of exogenous human tau in-transgenic mice. A, Hippocampal CA3 area, where we can easily evaluate tau localization in the mossy fiber axons (\#) and adjacent cell bodies of (A3 pyramidal neurons (*), was imaged for endogenous mouse tau, exogenous human tau, and both (total tau). Brain sections from WT (non-Tg) and P301L mutant $\mathrm{Tg}$ (P301L-Tg) mice were labeled with RTM47 (anti-mouse-tau shown in green in composite), anti-tauN (anti-total tau in blue), and tau12 (anti-human-tau in red). The signal intensity of each labeling was adjusted linearly to have a similar intensity in the axons. Scale bar, $20 \mu \mathrm{m}$. B, Distributions of mouse (red) and human tau (green) in the dentate gyrus of P301L-Tg mice. Only human tau immunoreactivity was apparent in the granule layer.

Quantification of mRNAs and proteins. Total RNA was isolated from the cortices of mouse brains using ISOGEN-LS (Nippon Gene), according to the manufacture's protocol. Extracted total RNA was purified using OligotexTM - dt $30<$ Super $>$ mRNA purification kit (Takara). Obtained mRNA was used for the synthesis of cDNA with Takara RNA PCR kit (AMV) (Takara). Subsequently, RT-PCR was performed with cDNA, Power SYBR Green PCR Master Mix (Thermo Fisher Scientific), and primers for the genes of interest (Table 1) on the 7500 Real-Time PCR system (Thermo Fisher Scientific). For relative quantification, levels of mRNA were normalized to that of mouse Gapdh. For the gene chip analysis, total RNA purified from three brains of 1 week or 2 weeks old mice was processed with the Ambion WT Expression Kit (Thermo Fisher Scientific) according to the manufacturers' instructions. cRNA was then fragmented, labeled, and hybridized to the Mouse Gene 1.0 ST Arrays using the Gene Chip WT Terminal Labeling and Hybridization Kit (Thermo Fisher Scientific) in GeneChip fluidics station 450 . Fluorescent signals were detected with the GeneChip scanner 3000-7 G. Images were analyzed with the GeneChip operating software (Thermo Fisher Scientific).

For protein analyses, mouse cortices were homogenized at room temperature in $\mathrm{O}+$ buffer: $62.5 \mathrm{~mm}$ Tris- $\mathrm{HCl}, \mathrm{pH} 6.8,10 \%(\mathrm{w} / \mathrm{v})$ glycerol,
5\% (v/v) 2-mercaptoethanol, 2.3\% (w/v) SDS, protease inhibitors (PMSF, DIFP, pepstatin, antipain, aprotinin, leupeptin, TLCK) and phosphatase inhibitors (NaF, $\beta$-glycerophosphate, $\mathrm{Na}_{3} \mathrm{VO}_{4}$, okadaic acid) (Planel et al., 2001). The homogenate was boiled for $5 \mathrm{~min}$ and subjected to centrifugation at $100,000 \times g$ for $15 \mathrm{~min}$. The supernatant was mixed with Laemmli sample buffer and subjected to SDS-PAGE. Proteins were separated on $10 \%$ acrylamide Tris/glycine gels and subjected to Western blotting with BD-anti-tau for total tau, anti-rodent tauN for mouse tau, tau12 for human tau, or DM1A for $\alpha$-tubulin. The blots were developed using an enhanced chemiluminescent detection (ECL) reagent (GE Healthcare), and the signals were analyzed with a LAS-4000 luminescent image analyzer systems (GE Healthcare). For quantification, the serial diluted samples of wild-type mouse brain cortices at 4 weeks old were used as a standard.

In situ hybridization. In situ hybridization of endogenous mouse tau and exogenous human tau mRNAs were performed using ViewRNA ISH Tissue Assay Kit (Thermo Fisher Scientific) according to the manufacture's instruction. Briefly, deparaffinized brain sections were subjected to the protease treatment for $10 \mathrm{~min}$, followed by the incubation with target probes for mouse tau mRNA (VB4-17162) and human tau mRNA 

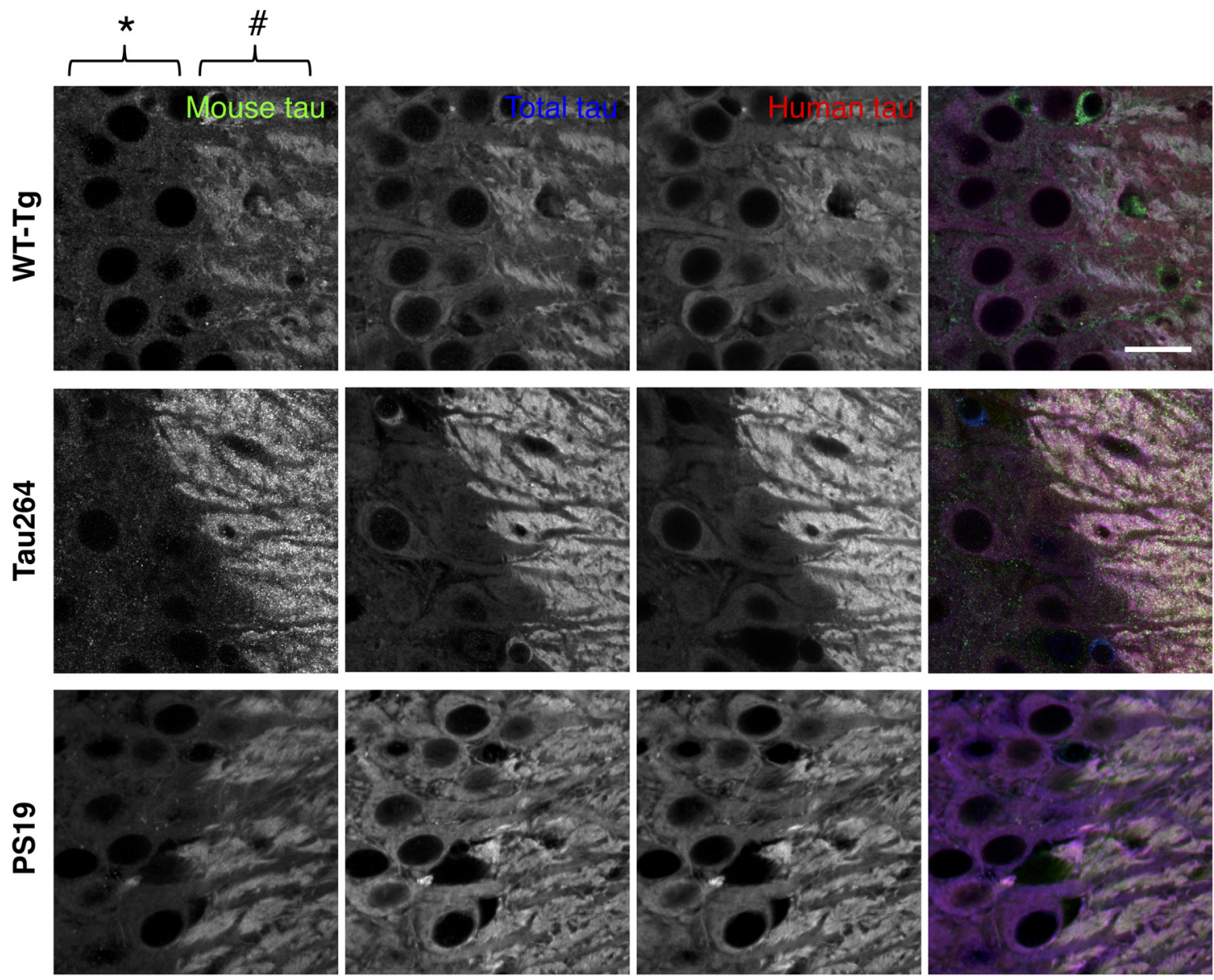

Figure 3. Abnormal somatodendritic localization of exogenous human tau in various transgenic mouse lines. Hippocampal CA3 was labeled for mouse, human, and total tau as in Figure $2 A$. Transgenic mice expressing high levels of WT human tau (WT-Tg), low levels of human tau (Tau264), and P301S mutant tau (PS19) were used. Mossy fiber axons and adjacent cell bodies of CA3 pyramidal neurons are indicated by \# and *, respectively. Scale bar, $20 \mu \mathrm{m}$.

(VA1-17145). Bound probes were visualized by probe 6-AP (blue) for mouse tau or probe 1-AP (red) for human tau mRNA, respectively. Then, the sections were counter stained with hematoxylin and embedded in ProLong Diamond Antifade Mounting medium (Thermo Fisher Scientific).

Experimental design and statistical analysis. Statistical analyses were performed on GraphPad Prism software. Power analysis was performed using $\mathrm{G}^{\star}$ Power with parameters taken from previous reports or similar experiments (Faul et al., 2007; Faul et al., 2009).

For the quantification of tau distribution in vivo, we measured fluorescence intensities of mouse (using RTM47) and human tau (with tau12) in the CA3 pyramidal layer and the striatum radiatum, for somatic and axonal signals, respectively, in the P301L-Tg and P301L-KI mouse brain sections. Somatic signals were measured around the nuclei of pyramidal cells, and axonal signals were quantified in the mossy fiber axons. The ratio of somatic and axonal signals were computed, and the averages were analyzed using two-way repeated-measures ANOVA with Sidak post hoc test to compare between the ratios of endogenous and exogenous tau in each animal model and between the animal models.

For the MT-binding assay, we measured and compared the levels of endogenous mouse tau and exogenous human tau in the unbound fraction in each mouse model using paired Student's $t$ test. Also, the levels of human tau in the unbound fraction were compared between the transgenic and knock-in mice using unpaired $t$ test.

To analyze the expression patterns of endogenous and exogenous proteins in mouse brains, we used regression analyses. We first measured the mRNA levels of tau, tubulins, and calmodulin kinase II (CaMKII) at different developmental stages. The levels were normalized to the average level at day 1 (for tau and tubulins) or day 70 (for CaMKII) after birth. The normalized levels were plotted against time in days after birth. We then tested whether each dataset can be fitted with a single exponential function:

$$
y=\operatorname{Span} \times e^{(-\mathrm{k} \times \mathrm{x})}+\text { Plateau }
$$

We also determined whether a single exponential function with shared slope and plateau can fit the data of tau with either those of tubulins or CaMKII. The latter analysis was supposed to provide us which expression pattern tau follows better. We also compared the span from the exponential curve, which is the difference from the initial value to the plateau value, for each animal. The span therefore reports the direction and magnitude of the change, such that a decrease and an increase in expression would result in positive and negative values, respectively. The mean values were then compared among different mouse proteins using repeated-measures ANOVA with the Tukey post hoc test or between endogenous and exogenous tau in each animal model using repeatedmeasures two-way ANOVA with Sidak test.

For the protein expression analysis, the ratio of the level at $\sim 10-13$ weeks over that at day 1 were computed for both endogenous and exogenous tau in transgenic (P301L-Tg) and knock-in mice. The values were compared using repeated-measures two-way ANOVA with Tukey post hoc test.

\section{Results}

To investigate the tissue distribution of tau, we recently raised anti-tau antibodies, which can distinguish endogenous mouse and exogenous human tau independently of the phosphorylation state, and demonstrated the detailed localization of endogenous mouse tau in nontransgenic (non-Tg) mouse brains (Kubo et al., 2019). As shown in Figure 1, our antibody demonstrates the 

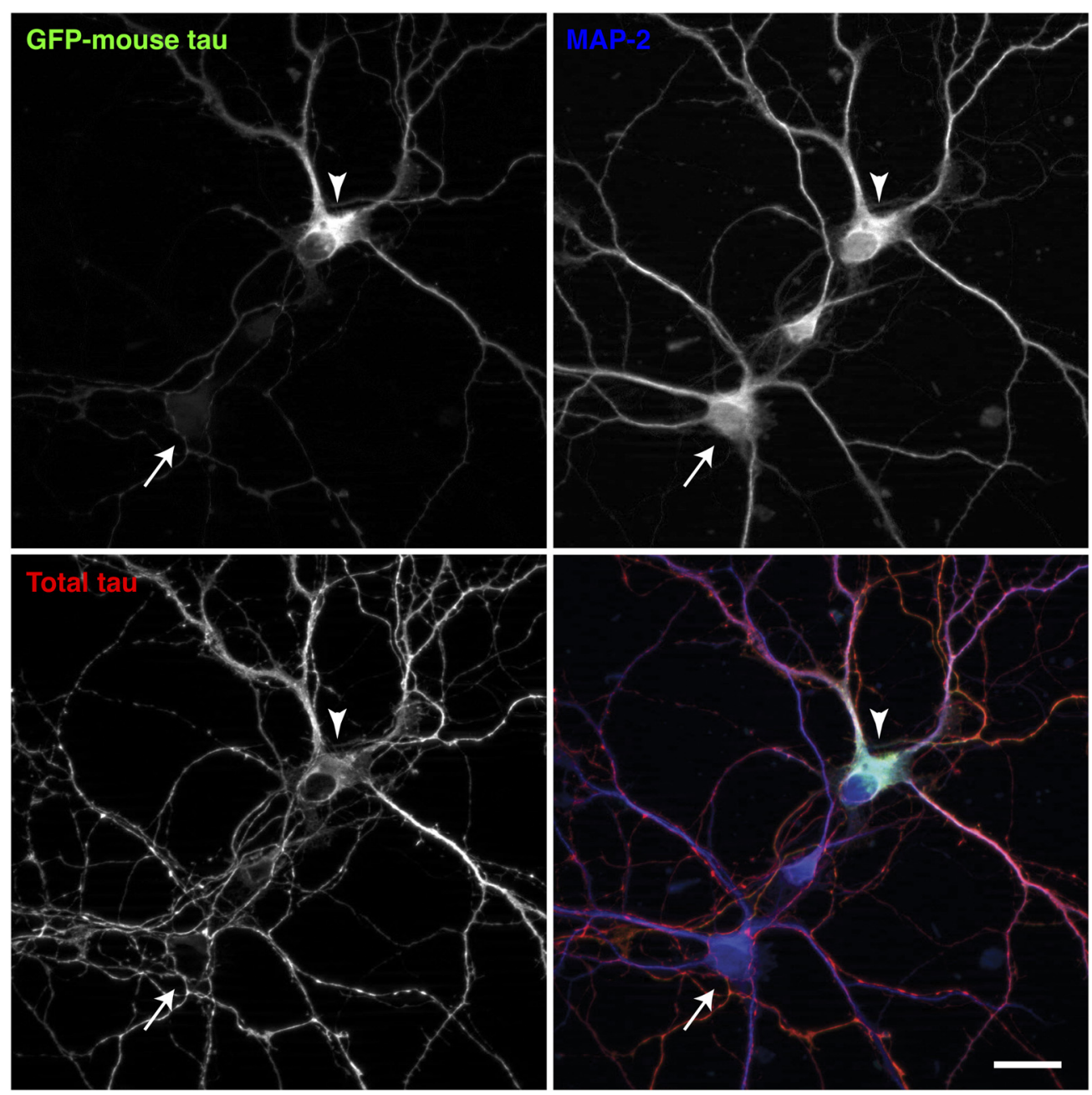

Figure 4. Mislocalization of exogenous mouse tau in mouse neurons in culture. Cultured mouse neurons from non-Tg mouse hippocampi were transfected at 7 DIV with GFP-tagged mouse tau, the expression of which is regulated by a CMV promoter. They were fixed at $14 \mathrm{DIV}$ and immunostained for total tau (red in the merged image) and MAP2 (in blue). The arrowhead indicates the soma of a neuron that expresses GFP-mouse tau, and the arrow indicates the soma of a nontransfected neuron. Scale bar, $20 \mu \mathrm{m}$.

axon-specific distribution of endogenous mouse tau in the hippocampus, particularly evident in the mossy fiber axons. Using these antibodies, here we investigated how somatodendritic tau emerges in mouse models of tauopathy.

We first examined the brains of P301L mutant (P301L-Tg) human tau Tg mice, in which human tau is expressed under the $\mathrm{Ca}^{2+} /$ CaMKII promoter (Kimura et al., 2010). In this mouse model, neuronal loss and tau pathology occur after the age of 20 months (Kimura et al., 2007, 2010). At the age of 6-12 months, which corresponds to a presymptomatic period, endogenous mouse tau, visualized by RTM47 mouse tau-specific antibody, was localized to axons such as mossy fibers in the brains of P301L-Tg (Fig. 2); this pattern was indistinguishable from that in non-Tg mice (Fig. 1). In contrast, exogenous human tau, labeled by human-specific tau 12 antibody, was localized not only to the axonal compartment but to the dendrites and cell bodies of CA3 pyramidal neurons (Fig. 2A) as well as in granule cells in the dentate gyrus (Fig. 2B).

The abnormal localization of exogenous human tau was not due to the pathogenic FTDP-17 mutation because similar aberrant localization of human tau was observed in the brains of wild-type human tau Tg (WTtau-Tg) mice (Fig. 3). Furthermore, the abnormal localization was not caused by the $3-5$-fold greater expression of human tau compared with endogenous tau in these mice (Kimura et al., 2010), as a mild but similar abnormal distribution of exogenous human tau was found in the brains of Tau264 mice (Fig. 3), in which the expression level of wild-type human tau is $\sim 10 \%$ of that of endogenous mouse tau (Umeda et al., 2013). Even at such low expression levels, the exogenous tau was still distributed in cell bodes and dendrites (Fig. 3). This abnormality was also observed for human tau expressed in the brains of PS19 mice, a tau-Tg line widely used for the tauopathy research (Fig. 3).

The abnormal distribution of exogenous tau could be due to the expression of human tau in mouse neurons. To investigate this cross-species issue, we transfected cultured mouse neurons with GFP-tagged mouse tau, the expression of which is driven by the CMV promoter. The exogenous GFP-tagged mouse tau appeared to be mislocalized to the soma and dendrites, whereas endogenous mouse tau was properly localized to MAP2-negative axons (Fig. 4), indicating that the mislocalization does not result from the species difference.

We also analyzed the distribution of tau in the brains of P301Ltau knock-in (P301L-KI) mice, in which exogenous human tau replaces endogenous mouse tau and is expressed under the genuine tau promoter. Surprisingly, the exogenous P301L mutant human tau showed a normal axonal distribution, which was particularly apparent by comparison with endogenous 

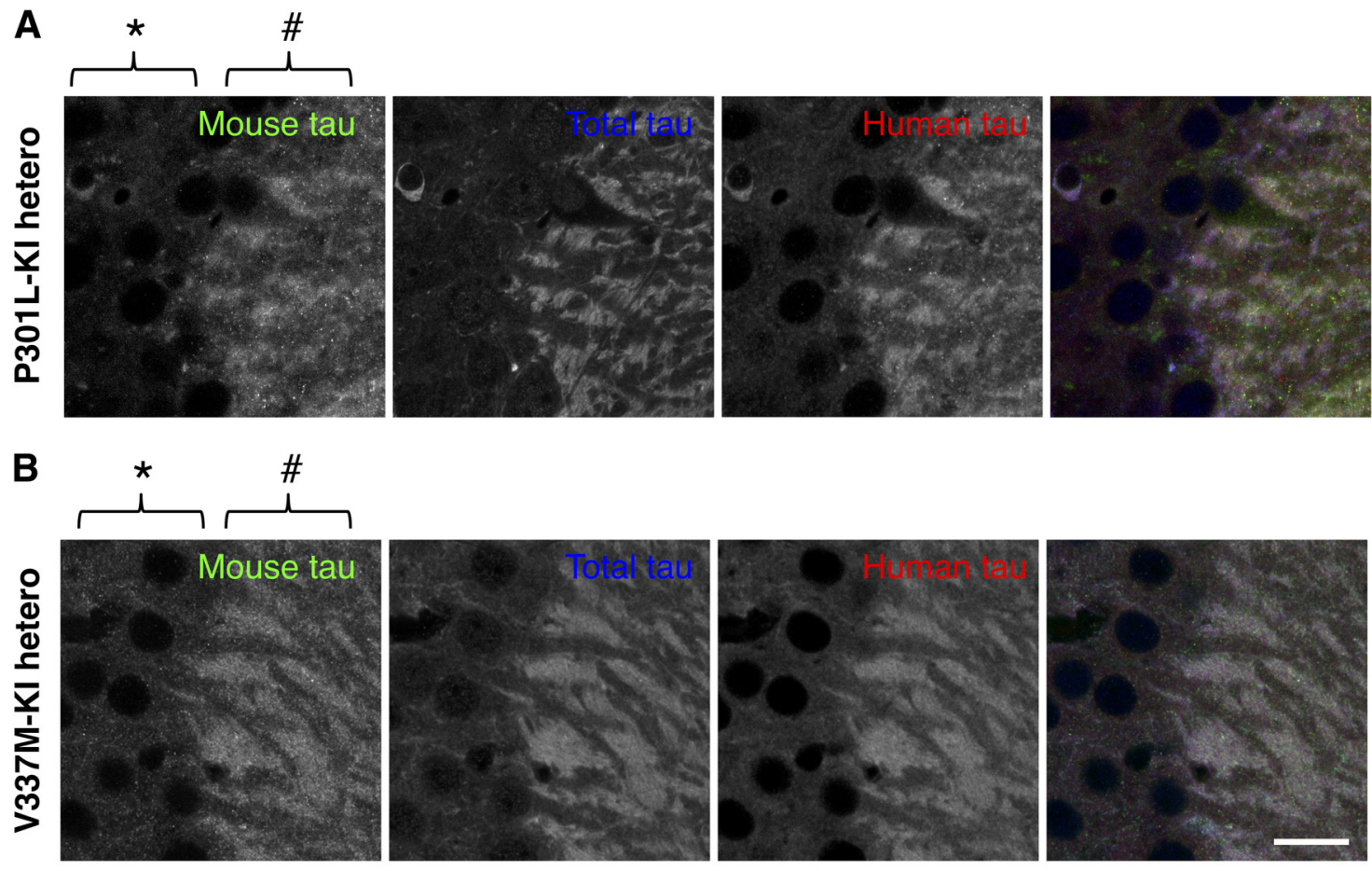

C

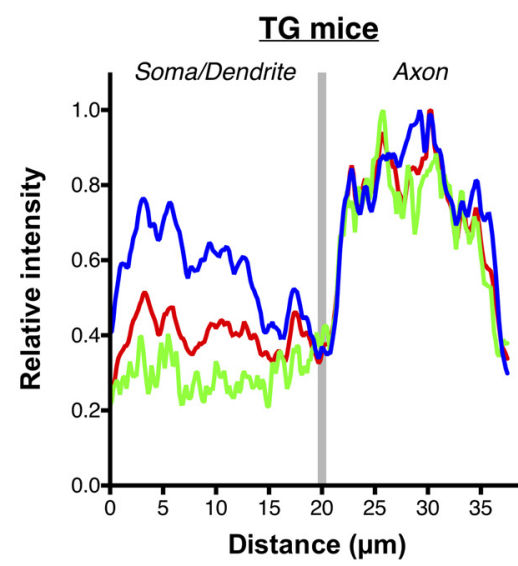

KI mice (hetero)

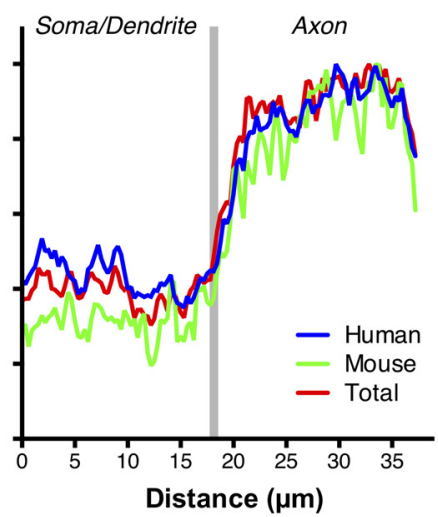

D
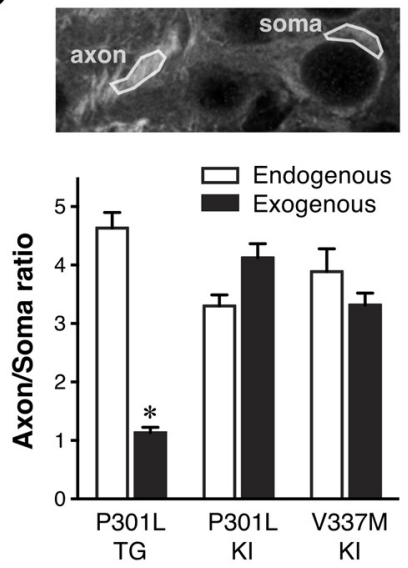

Figure 5. Normal axonal localization of exogenous human tau in P301L tau knock-in mice. A, Mouse and human tau in the hippocampal CA3 of heterozygous P301L-KI mice were immunolabeled with anti-mouse tau (RTM47 shown in green), anti-total tau (tauN in blue), and anti-human tau (tau12 in red). It should be noted that, unlike in P301L-Tg mice, both mouse and human tau were virtually absent in the somata (*) but abundant in the axons (\#). Scale bar, $20 \mu \mathrm{m}$. B. Mouse and human tau in the hippocampal CA3 of heterozygous V337M-KI mice immunolabeled as in A. Scale bar, $20 \mu \mathrm{m}$. C, Normalized intensity profiles of RTM47, anti-tauN, and tau12 in P301L-Tg and V337M-KI mice along lines drawn from the CA3 pyramidal cell layer (Soma/Dendrite) to mossy fiber (Axon) are shown. D, To quantify the difference in tau localization between $\mathrm{Tg}$ and $\mathrm{KI}$ mice, the axon/soma ratio of fluorescence intensity was computed and compared (see Materials and Methods). Endogenous mouse tau exhibited high ratios indicating its enrichment in the axon in both mouse models. In contrast, the exogenous human tau in P301L-Tg mice showed significantly lower ratios than those of endogenous mouse tau in the same animals $\left(t_{(10)}=13.87, p<0.0001\right.$ using repeated-measures two-way ANOVA and Sidak test $)$ and exogenous human tau in P301L-KI mice $\left(t_{(20)}=\right.$ $7.012, p<0.0001)$ and in V337M-KI mice $\left(t_{(20)}=5.287, p=0.0002\right)$.

mouse tau in heterozygous mice (Fig. 5A). The normal axonal localization is not mutation-specific, as V337Mtau also showed axonal localization in V337M-KI mice (Fig. 5B). Line scan analysis and the comparison of axon/soma ratios demonstrated that exogenous tau in heterozygous KI mice exhibits localization indistinguishable to that of endogenous tau, whereas human tau in P301L-Tg mice shows abnormal somatodendritic localizations (Fig. $5 C, D$ ). Thus, the abnormal distribution of exogenous tau in $\mathrm{Tg}$ mice in dendrite and somata is not due to the species difference nor mutations, but rather the regulatory mechanisms for tau expression.

We recently reported the localization of endogenous tau in the mouse brain at superresolution $(\sim 50 \mathrm{~nm})$ using STED micros- copy (Kubo et al., 2019). With the N-terminally binding antibody (anti-tauN), we showed that tau was discontinuously, but not uniformly, distributed in the axons in the CA1 stratum radiatum (Fig. 6A-C) and in the mossy fiber axons of the hippocampus (Fig. 6D,E). The mossy fiber axons with a diameter of 200-500 $\mathrm{nm}$ are typically packed with several MTs observable with electron microscopy (Amaral et al., 2007). Despite this high density of MTs, tau labeling did not appear fibrous but punctate. This unique labeling was reproduced using the RTM 38 antibody, the epitope of which differs from that of anti-tauN and is in the C-terminal region of tau (Fig. 6F). In contrast, in P301L-Tg mice, exogenous tau in the mossy fiber axons distributed continuously and appeared fibrous, whereas endogenous mouse tau in the 
A

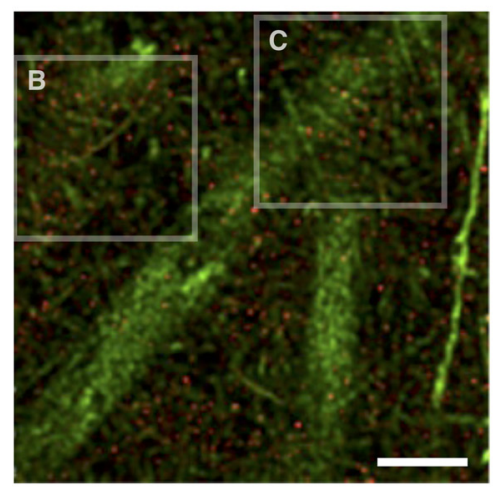

D

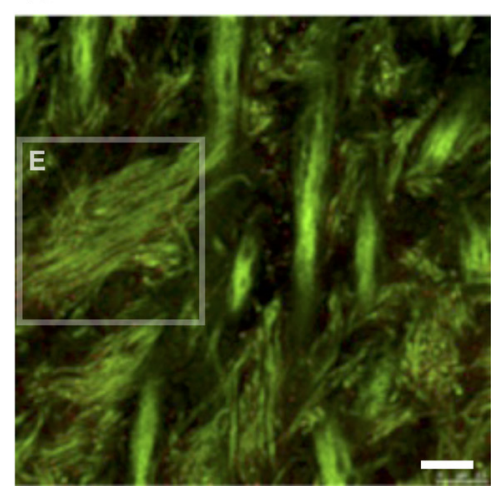

B

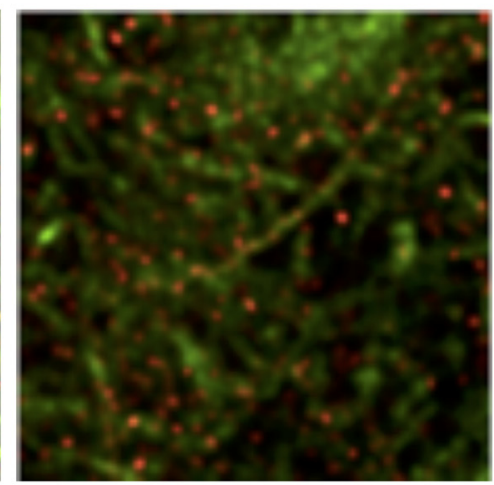

E

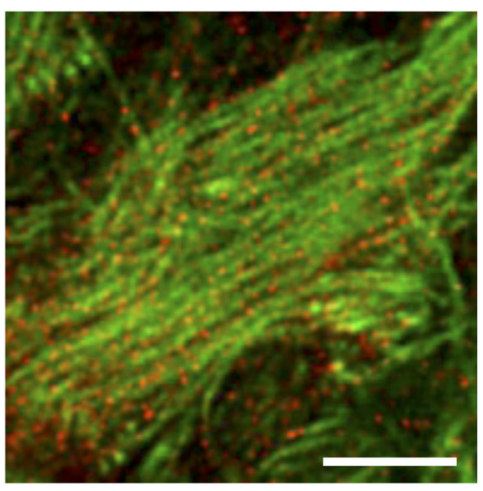

C

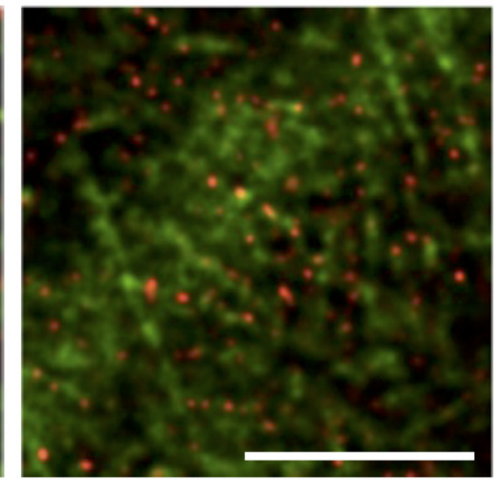

F

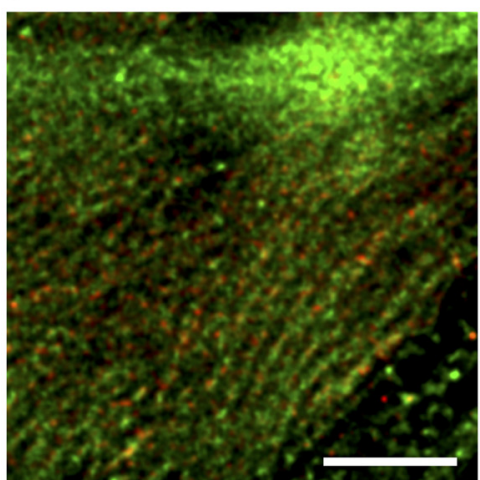

Figure 6. High-resolution imaging of tau in the hippocampal region of non-Tg mouse brains. $\boldsymbol{A}$-C, Hippocampal CA1 in non-Tg mice labeled with DM1A (green) and anti-tauN (red) and imaged using STED. The boxes in $\boldsymbol{A}$ are the areas that are magnified in $\boldsymbol{B}$ and $\boldsymbol{C}$. In CA1, tau is discontinuously localized in axons. D, Hippocampal CA3 in non-Tg mice labeled with DM1A (green) and anti-tauN (red). The box in the left image is the area that is magnified in $\boldsymbol{E}$. Punctate tau labeling was observed on mossy fiber axons but was not found in the apical dendrites of pyramidal neurons in area $(A 3$. $\boldsymbol{E}$, Magnified image of CA3 labeled with DM1A (green) and tauN (red). $\boldsymbol{F}$, Magnified image of CA3 labeled with DM1A (green) and RTM38 (red). Scale bars, $2.5 \mu$ m.

same animals exhibited the punctate labeling (Fig. $7 A, B$ ). In contrast, exogenous human tau exhibited the punctate pattern in $\mathrm{KI}$ mouse brains (Fig. 7C). These results indicate that the binding of tau to axonal MT is also disrupted in the $\mathrm{Tg}$ mice but not in $\mathrm{KI}$ mice.

We therefore examined biochemically whether the mislocalization of exogenous tau in tau-Tg mice is associated with abnormal MT-binding. In the brains of non-Tg mice, the proportion of MT-unbound tau was $23.7 \pm 1.7 \%$ (Fig. $8 A, B$ ). By contrast, in P301L-Tg mice, the proportion of MT-unbound exogenous human tau $(64.8 \pm 1.4 \%)$ was significantly higher than that of MTunbound endogenous mouse tau $(24.9 \pm 0.5 \%)\left(q_{(9)}=35.24\right.$, $p<0.0001$ using Tukey test after ANOVA, $F_{(2,9)}=427.7, p<$ $0.0001)$ or MT-unbound tau in non-Tg mice $\left(q_{(9)}=36.37, p<\right.$ 0.0001 ) (Fig. $8 A, B$ ). There was no difference in MT binding of endogenous tau between the non-Tg and P301L-Tg mouse brains $\left(q_{(9)}=1.128, p=0.7137\right)$. Thus, in Tg mice, only exogenous tau is poorly bound to MTs. We then analyzed the MT binding of exogenous tau in heterozygous KI mice, in which exogenous human tau was localized to the axon in the punctate manner. In these animals, the majority of exogenous tau was found MT bound, as was the majority of endogenous tau in non-Tg mice (Fig. $8 C-F$ ). It should be noted that the levels of MT-bound tau depend on how well MTs are preserved during an experiment, such that they differ significantly across experiments. There were no significant differences among the unbound fractions in each model $\left(F_{(2,8)}=0.9127, p=0.4395\right.$ for V337M-KI, and $F_{(2,9)}=$ 3.879, $p=0.0610$ for P301L-KI using ANOVA). These results suggest that exogenous human tau can exhibit a normal axonal distribution and MT-binding if it is expressed under the tau promoter regardless of mutation.

Because our data so far suggest that the ectopic expression of exogenous tau results in the mislocalization as well as abnormal binding to MTs, we investigated how the expression of normal tau and axonal localization occur. First, we investigated the timing when the axonal localization of tau is established during brain development in non-Tg mice. Double staining with RTM38 antitau and anti-MAP2N antibodies showed that tau remains in the cell body and colocalized with MAP2 at day 7 after birth (Fig. 9A). However, at day 14, somatic tau signals were greatly diminished, and tau and MAP2 exhibited discrete distributions in the axon and somatodendrites, respectively, as in adult animals (Fig. 9B). Thus, axonal distribution of tau completes between 7 and $14 \mathrm{~d}$ after birth. We next examined the distribution of tau in the brains of P301L-Tg mice at this developmental stage. Interestingly, somatodendritic localization of exogenous tau was maintained even at P14 (Fig. 9C), whereas endogenous tau was properly localized to the axon. A similar abnormal distribution of exogenous tau was found in cultured hippocampal neurons from embryos obtained by crossbreeding non-Tg and heterozygous P301L-Tg mice. Resulting mixed neurons derived from either non-Tg or Tg embryos could be distinguished based on their immunoreactivity to the human-tau-specific taul2 antibody. In $\mathrm{Tg}$ and non-Tg neurons, endogenous tau was detected in the cell bodies at 3 DIV (Fig. 9D) but became localized to the axon at 14 DIV (Fig. 9E), as previously reported (Deshpande et al., 2008). Exogenous human tau was also found in the cell bodies at 3 DIV (Fig. 9D), but this somatic expression was maintained 
A

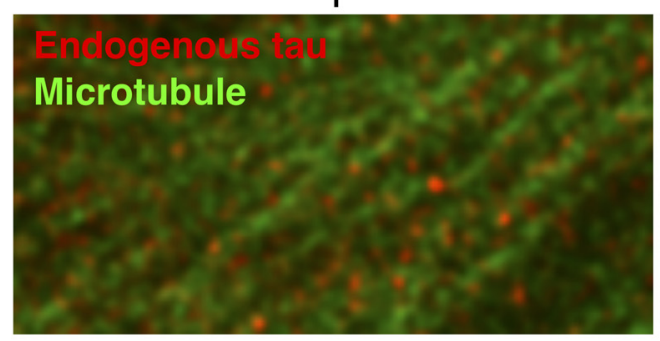

B
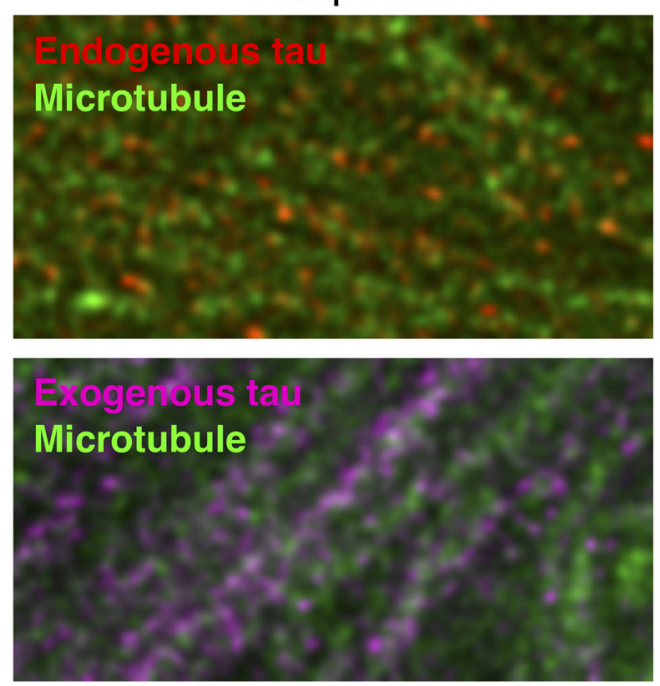

C

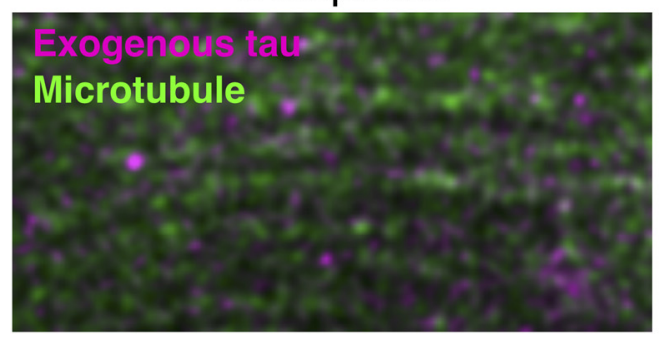

Tau

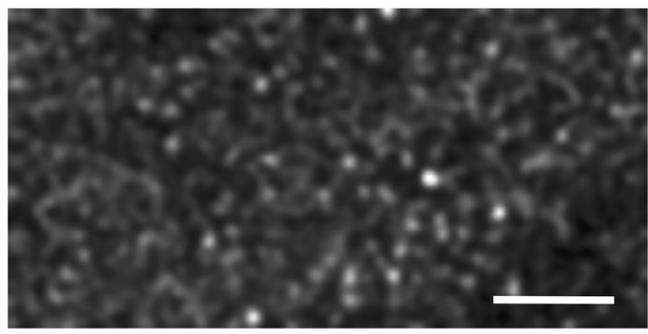

Tau
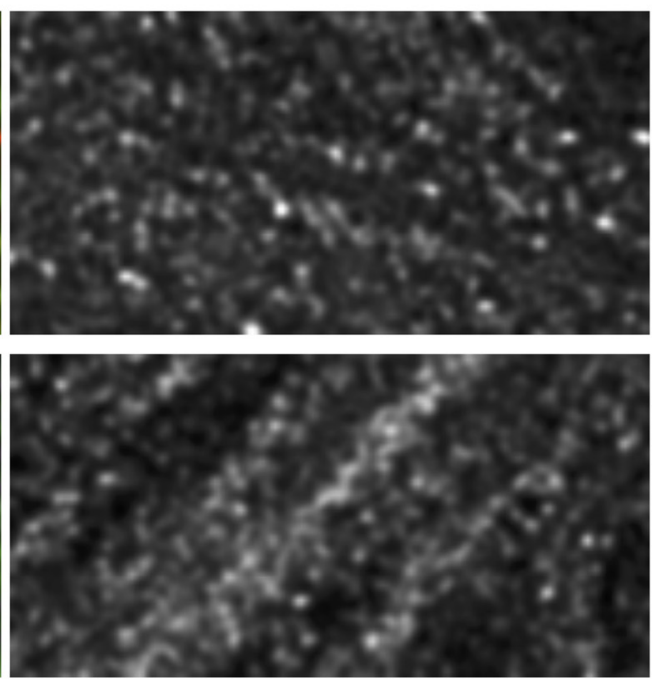

Tau

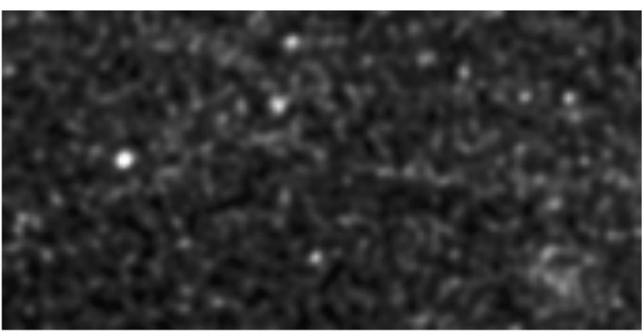

Figure 7. High-resolution imaging of endogenous and exogenous tau in the hippocampi of $\operatorname{Tg}$ and KI mouse brains, Mossy fibers of non- $\operatorname{Tg}(\boldsymbol{A}), \mathrm{P301L}-\mathrm{Tg}(\boldsymbol{B})$, and heterozygous V337M-KI ( $\boldsymbol{C}$ ) mice were labeled with DM1A (green) and anti-RtauN (endogenous tau, red) or RTM49 (human tau, magenta) and observed using STED microscopy. Merged views (left) and tau signal (right) were shown. Exogenous tau in $\mathrm{Tg}$ mice, but not in KI mice, showed a more continuous localization pattern. Scale bar, $1 \mu \mathrm{m}$.

even at 14 DIV in Tg neurons (Fig. 9E). Therefore, unlike endogenous tau, exogenous human tau, the expression of which is driven by the ectopic CaMKII promoter, maintains its somatodendritic localization throughout development and into adulthood in Tg mice.

Because the promoter of tau expression may dictate its localization in neurons, we investigated how the expression of endogenous tau is regulated by the genuine tau promoter and how the expression of exogenous tau differs in $\mathrm{Tg}$ mouse brains using qRT-PCR. First, the expression of endogenous tau-mRNA (Mapt) was quantified in non-Tg mouse cortices at different ages. The level of tau mRNA was highest in the neonatal stage and drastically decreased during the first and second week after birth (Fig. 10A), around the same time that the axonal distribution of tau was completed (Fig. 9B). After 4 weeks, the expression became stabilized at low levels (Fig. 10A). Interestingly, we also found that mRNA levels of $\alpha$-tubulin (Tuba1), $\beta$-tubulin (Tubb1), MAP-2 (Map2), and MAP-1B (Map1b) were regulated in a similar fashion (Fig. 10A, Table 2). By contrast, the expression of CaMKII (Camk2a), of which promoter was used for the P301L-Tg mouse, exhibited an inverse pattern to that of Mapt. To analyze and compare these patterns quantitatively, we fitted each dataset with a single exponential function (see Experimental Procedures). We found that the data of Mapt, Tuba1, Tubb1, Map2, Map 1b, and neurotrophin-3 (Ntf3) can be fitted well with exponential decay functions, whereas that of Camk2a fits better with an exponential growth function. Particularly, the decay curves for Mapt, Tubal, and Tubb1 had similar parameters, which were statistically indifferent $\left(F_{(6,69)}=0.1053, p=0.99\right)$. To compare these profiles with that of Camk2a, we extracted the span parameter, which reflects the direction and the magnitude of the change in the expression level, from each exponential function (Fig. 10B, 
A

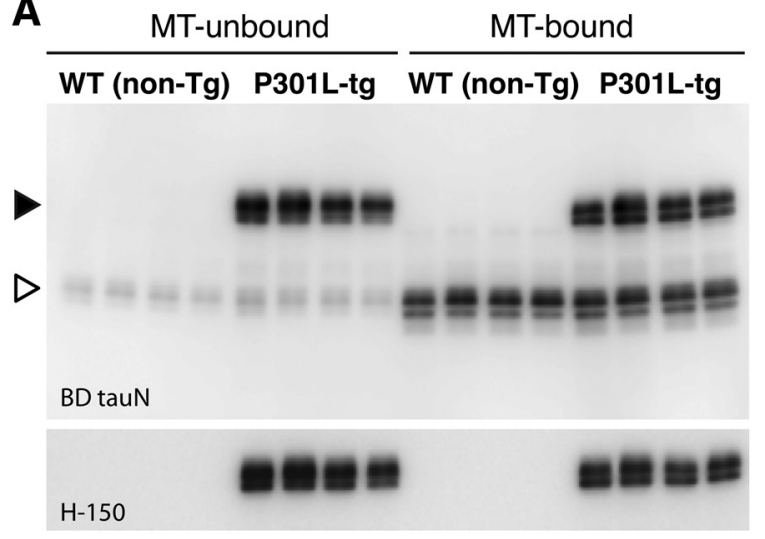

C

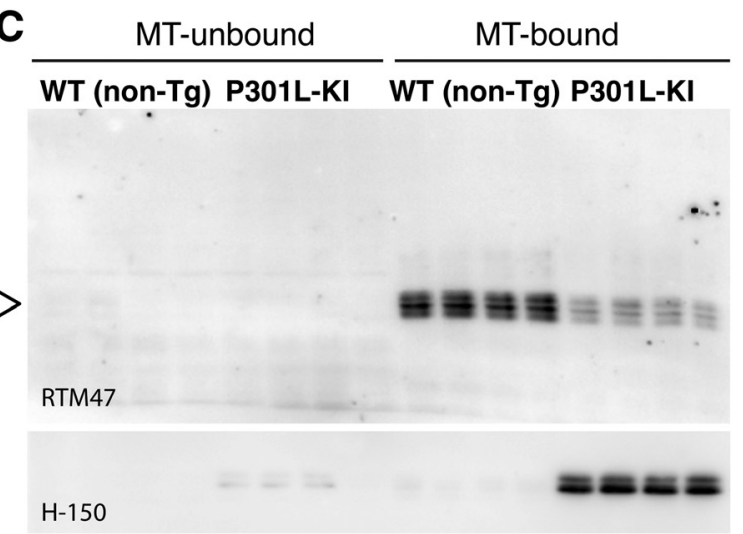

E

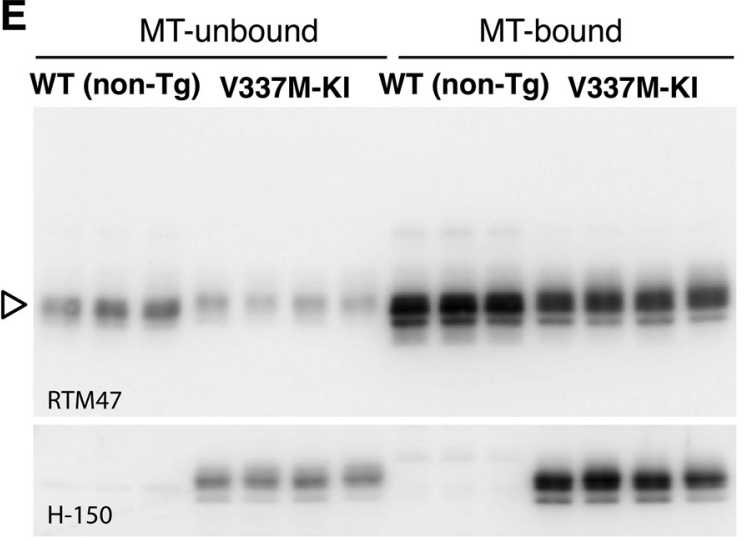

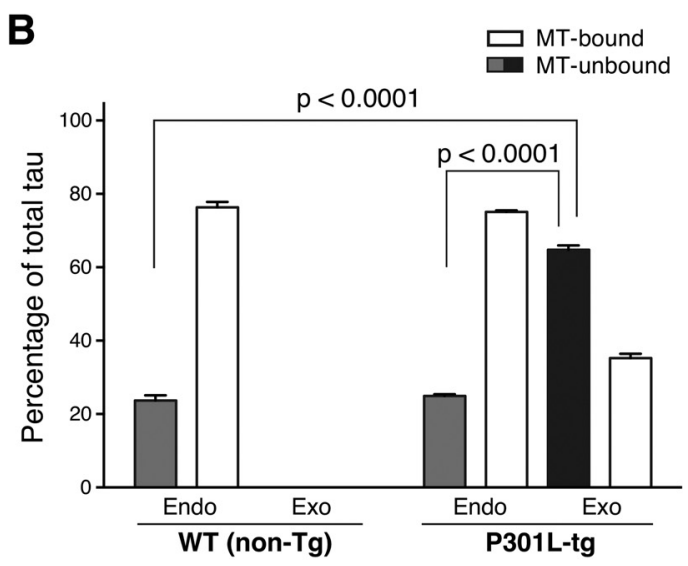

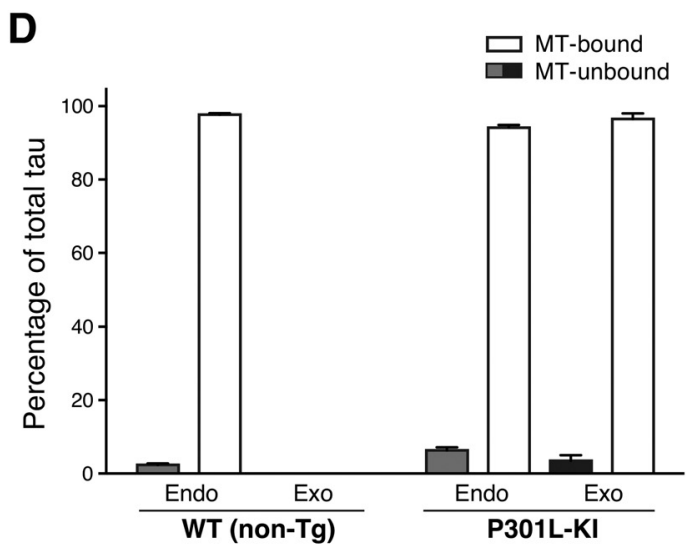

$\mathbf{F}$

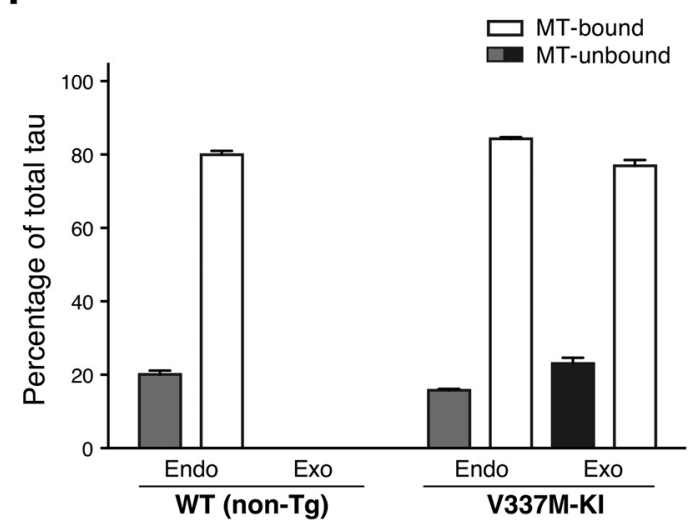

Figure 8. MT binding of human tau in Kl and Tg mice. MT-bound and NT-unbound tau were prepared from adult non-Tg and P301L-Tg (A), P301L KI (C), or V337M-KI mouse brains (E) as described in the Materials and Methods and detected in Western blotting using anti-total tau (BD anti-tau), anti-human tau (H-150), or anti-mouse tau (RTM47) antibodies. Human and mouse tau in Tg mouse brains are indicated by closed and open arrowheads, respectively. Samples in a single gel were prepared in a single experiment, and multiple samples prepared from different animals are shown. Immunoreactive bands of interest were quantified using known amounts of recombinant tau as standards. The proportions of MT-unbound (solid) and MT-bound (open) endogenous (endo) and exogenous (exo) tau are shown in $\boldsymbol{B}, \boldsymbol{D}$, and $\boldsymbol{F}$ for $\boldsymbol{A}, \boldsymbol{C}$, and $\boldsymbol{E}$, respectively. The averages of the unbound fraction were compared in each experiment using two-way ANOVA with Sidak post hoc test because the preservation of MTs and the level of MT-bound tau differ in every experiment. We therefore made comparisons only within each experimental set. The level of unbound human tau was significantly higher than that of endogenous tau in the Tg mice and in WT (non-Tg) mice $\left(F_{(2,9)}=427.7, p<0.0001\right)$. By contrast, there was no significant difference in the levels of unbound tau for KI mice $\left(F_{(2,8)}=0.9127, p=0.4395\right.$ for $\boldsymbol{D} ; F_{(2,9)}=3.879, p=0.061$ for $\left.\boldsymbol{F}\right)$.

Table 2). As expected, spans of Mapt and Tubal showed large positive values $(1.02 \pm 0.16$ and $1.06 \pm 0.05$, respectively), reflecting that they decay over time, whereas the span for Camk2a was negative $(-1.02 \pm 0.04)$ (Fig. 10C). The overall difference of these values was statistically significant $\left(F_{(1.202,3.605)}=168.1, p=\right.$ $0.0003)$, and there were significant differences between Mapt and Camk2a $(p=0.0025)$ and between Tuba1 and Camk2a $(p<$ $0.0001)$, but not between Mapt and Tubal ( $p=0.95)$.
The large reduction of tau mRNA expression between 1 and 2 weeks after birth was also confirmed in our gene-chip analysis, such that tau (Mapt) was one of the genes that showed a $>2$-fold decrease during this period (Fig. 11A). Despite the large changes in mRNA expression during the perinatal development, protein levels of total tau including both three- and four-repeat tau were relatively constant (Fig. 11B). We also performed in situ hybridization and verified the developmental regulation of tau mRNA 
A
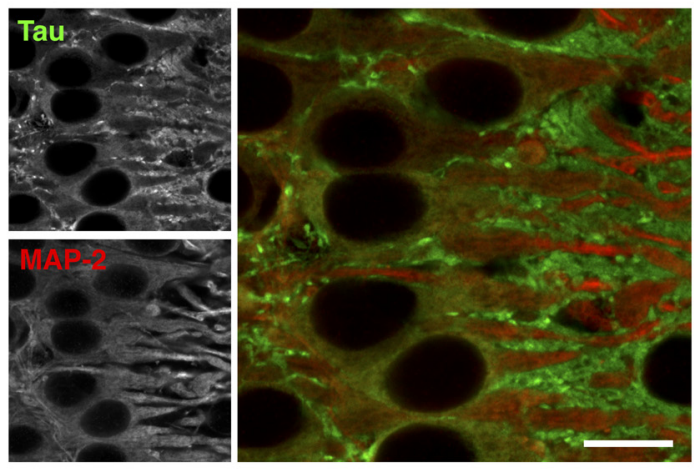

C
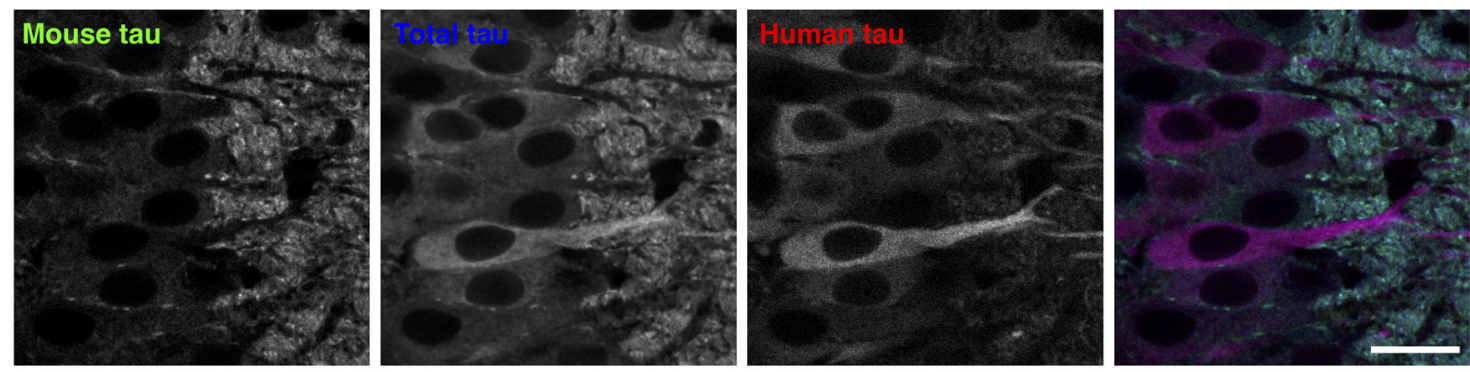

D
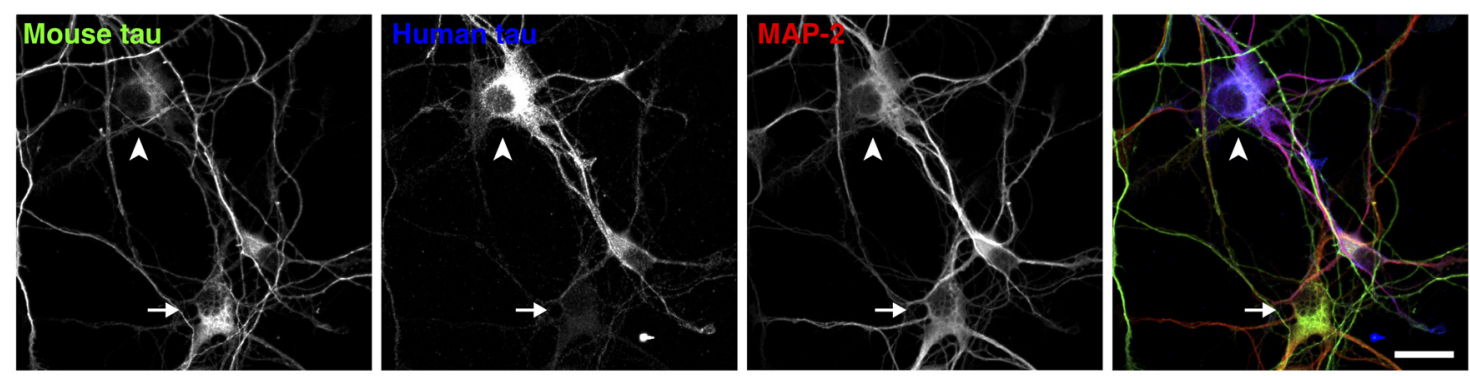

\section{$\mathbf{E}$}
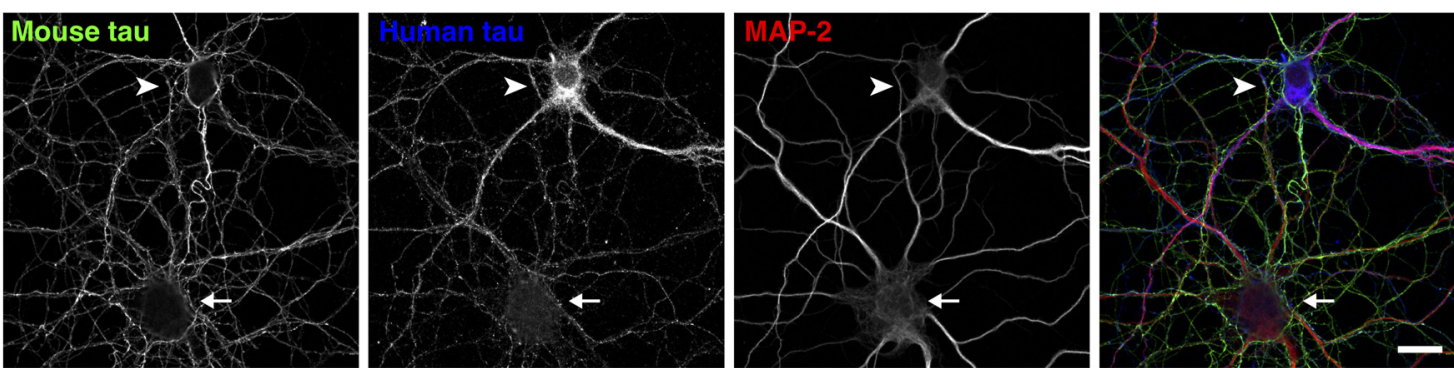

Figure 9. Localization of tau in P301L-Tg mice during development. $A$, Brain sections from P7 non-Tg mice were subjected to immunostaining for tau (anti-tauN, green) and MAP2 (HM2, red). There were some overlapping signals of tau and MAP-2 in the somata. Scale bar, $20 \mu \mathrm{m}$. B, Brain sections from P14 non-Tg mice were immunostained for tau (anti-tauN, green) and MAP2 (HM2, red), which exhibited interdigitated patterns. Scale bar, $20 \mu \mathrm{m}$. C, Brain sections from P14 P301L-Tg mice were subjected to immunostaining for mouse tau (anti-RtauN, green), total tau (tauN, blue), and human tau (Tau12, red). Scale bar, $20 \mu \mathrm{m} . \boldsymbol{D}, E$, Mixed primary cultures of hippocampal neurons from the brains of both non-Tg and P301L-Tg mice were immunostained with anti-mouse tau (RTM47, green), anti-human tau (tau12, blue), and anti-MAP2N (red) at 3 DIV (D) and 14 DIV (E). The arrowheads indicate cells that were derived from P301L-Tg mice and the arrows indicate cells from non-Tg mice. Scale bars, $20 \mu \mathrm{m}$.

(Fig. 11C,D). The results showed that the tau transcripts were readily detectable in the perikarya of CA3 pyramidal neurons in the newborn hippocampus (Fig. 11C), whereas they were at very low levels, albeit detectable, in adult (Fig. 11D). It should also be noted that mRNA signals were very scarce outside of the pyramidal layer in the CA3 area at both time points. These results suggest that the mRNA expression of endogenous tau is highly active during early development, which presumably results in the constant expression of tau proteins.

The expression profile of Camk2a indicated that the expression of human tau in the P301L-Tg mice persists beyond the perinatal period, thereby resulting in the sustained somatodendritic localization of human tau. To test this, we examined the mRNA and protein expression of human tau in this animal 
A
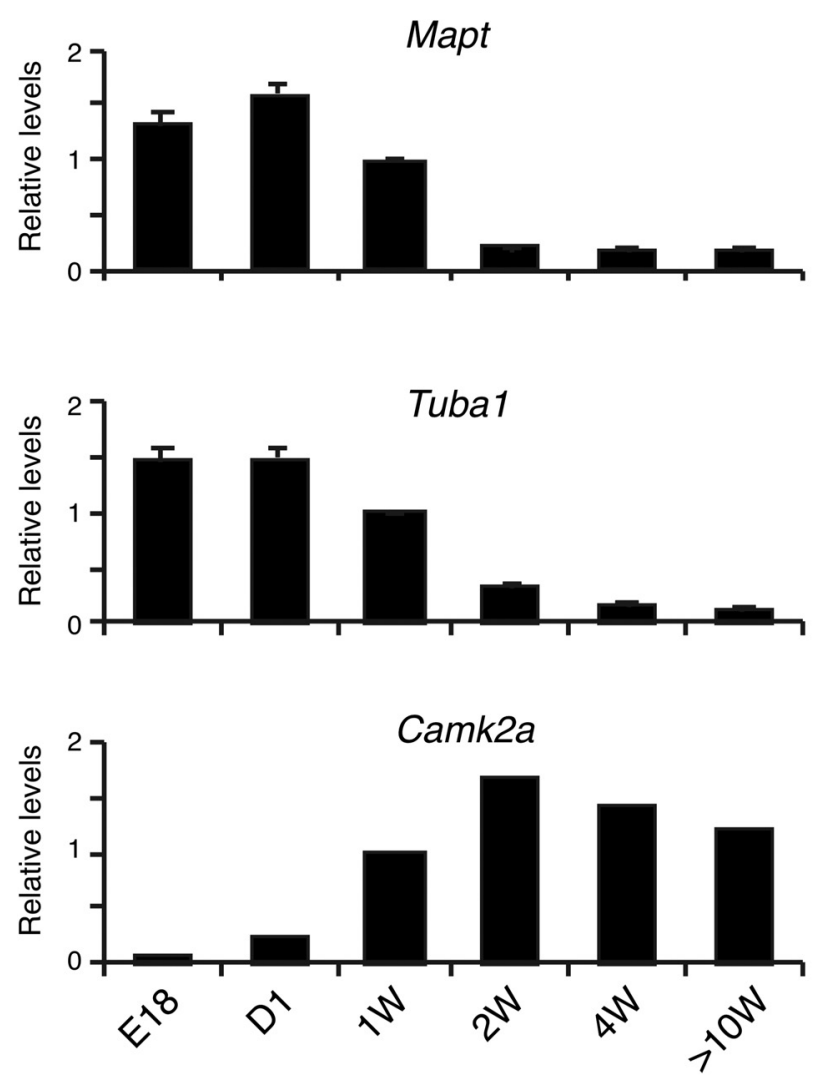

B

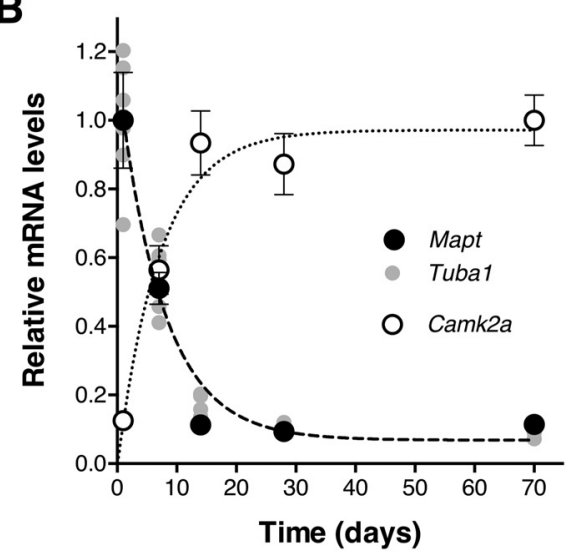

C

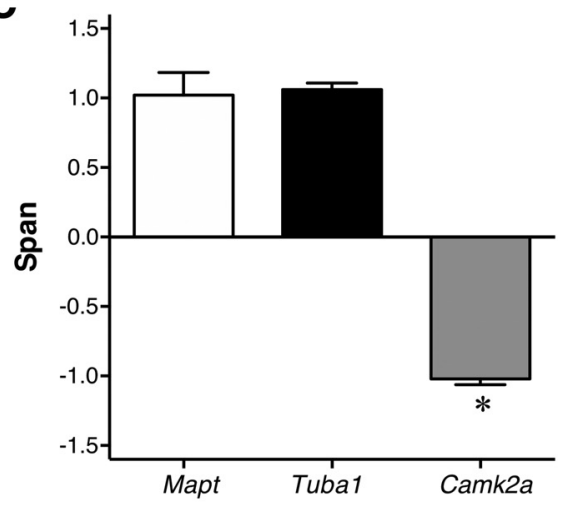

Figure 10. Developmental expression of endogenous tau in the mouse brain. $A$, Relative levels of mRNA of tau (Mapt),-tubulin (Tuba1), and CaMKII (Camk2a) at E18, $1 \mathrm{~d}$ (D1), 1 week (1W), 2 weeks (2W), 4 weeks (4W), and 10-13 weeks (10W) after birth in the cerebral cortices of non-Tg mice were quantified using qRT-PCR. The levels were normalized to that at 1 week for each gene. B, Data in A were analyzed using nonlinear regression with single exponential curves (top). $\boldsymbol{C}$, The span of the function in B (see Materials and Methods) was obtained from each animal, and the averages were compared among the three genes using repeated-measures ANOVA with Tukey test $\left(\mathrm{F}_{(1.202,3.605)}=168.1, p=0.0003\right)$.

Table 2. Developmental expression of tau and other genes in the mouse cortex

\begin{tabular}{lc}
\hline Gene & \multicolumn{1}{l}{ Span } \\
\hline Tubulins and MAPs & \\
$\quad$ Mapt & $1.02 \pm 0.16$ \\
Tuba1 & $1.06 \pm 0.05$ \\
Tubb1 & $1.08 \pm 0.07$ \\
Map2 & $0.82 \pm 0.02$ \\
$\quad$ Map1b & $0.74 \pm 0.12$ \\
Other genes & \\
$\quad$ Camk2a & $-1.02 \pm 0.04^{*}$ \\
Ntf3 & $1.05 \pm 0.12$ \\
\hline${ }_{p}<0.0001$ compared with all other genes $\left(F_{(620)}=66.91\right)$. &
\end{tabular}

model. As shown in Figure 12A, the level of exogenous tau mRNA in the Tg mouse was lowest at day 1 and continuously increased toward adulthood, that is consistent with the Camk2a mRNA expression profile (Fig. 12A). In contrast, the expression of human tau mRNA in KI mice was at the highest at day 1 and then steeply decreased afterward like endogenous tau (Fig. 12A). We also fitted these data with exponential functions and computed the spans. The results clearly show that the expression of human tau in the Tg mice is inversely regulated compared with that of endogenous tau (Fig. 12B). Double-labeling in situ hybridization also revealed very high expression of human mRNA in adulttransgenic mice (PS19), whereas endogenous tau mRNA was barely detectable in the same neurons (Fig. 12C). Concomitantly, the amount of human tau protein in $\mathrm{Tg}$ mice also showed a significant age-dependent increase $\left(t_{(7)}=12.89, p<0.0001\right.$, two- way ANOVA with Sidak post hoc test), whereas that in KI mice was relatively constant $\left(t_{(7)}=2.238, p=0.12\right.$ ) (Fig. $\left.12 D-F\right)$. Together, these data suggest that excess expression of tau mRNA in mature neurons results in the mislocalization of tau protein to the somatodendritic compartment and abnormal MT binding.

We further investigated whether the mislocalization is critical in the development of tau pathology in these animal models. In the brains of aged PS19, the phospho-specific AT8 antibody strongly labeled punctate and filamentous structures in the somata in a subset of CA1 pyramidal cells (Fig. 13), indicating the formation of tau inclusions. Interestingly, we were able to detect human tau in these inclusions, but endogenous mouse tau was only faintly detected. Therefore, the somatodendritic inclusions in the brains of PS19 consist mostly of exogenously expressed human tau, which mislocalizes to the soma and dendrites. In contrast, the P301L KI mice did not exhibit any tau pathology even at $>18$ months of age, and even when they were crossbred with APP-Tg mice (Fig. 14), indicating that the mutation per se is not sufficient to results in tau inclusions. These results suggest that tau mislocalization to the soma and dendrites is necessary for the abnormal phosphorylation and the formation of inclusions.

\section{Discussion}

Previous studies of tau focused mostly on abnormal aggregated tau, and those investigating normal tau in vivo have been scarce (Iqbal et al., 2016). The major issue was the lack of immunological reagents sensitive enough to detect non-aggregated tau in vivo, as well as those specific for human and mouse tau individ- 
A

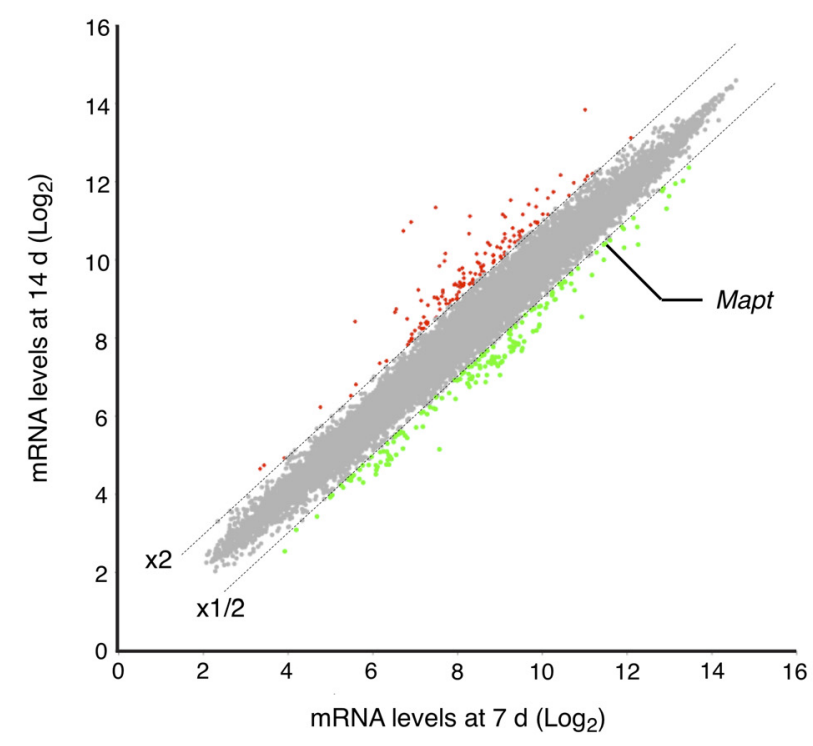

C
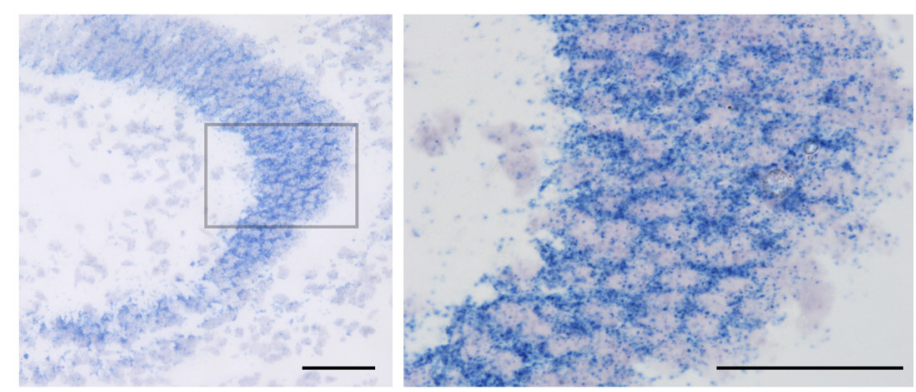

B
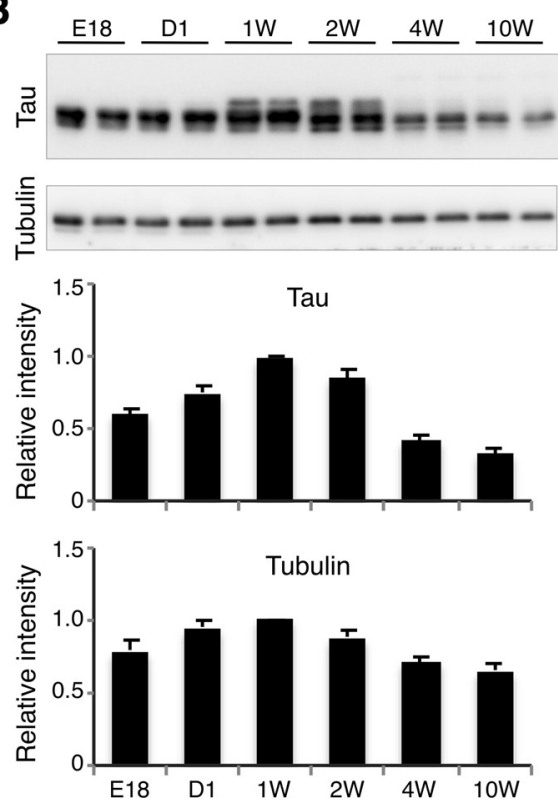

D

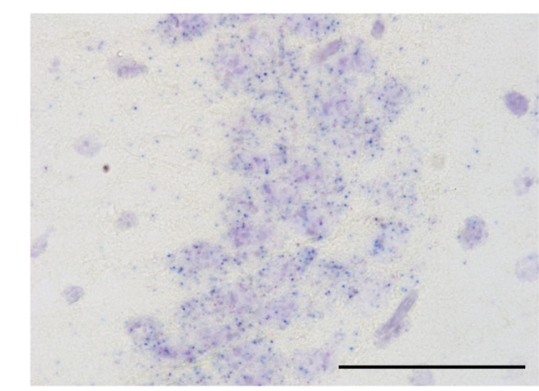

Figure 11. Transcription, translation, and mRNA distribution of mouse tau. $A$, Microarray analysis and comparisons of cortical transcripts between 1 and 2 weeks after birth. The dashed lines indicate either the twofold increase or decrease from the levels at 1 week. Tau (Mapt) was identified as one of the genes that exhibited significant decreases. $\boldsymbol{B}$, Protein levels of tau and $\alpha$-tubulin. Top, Western blots of tau and tubulin at E18, 1d (D1), 1 week (1W), 2 weeks (2W), 4 weeks (4W), and $>10$ W using anti-tauN and tubulin antibodies. Duplicates are shown for each time point. Bottom shows the results of quantitation as relative levels to that at 1 week. $C$, In situ hybridization of tau mRNA in the neonatal mouse brain. Brain sections from new born pups were subjected to in situ hybridization for endogenous tau mRNA (blue dots) using the ViewRNA method. The left image shows the hippocampal CA3 area. The box indicates the region shown in the right image at a higher magnification. Signals were found mostly in the pyramidal layer, but were very scarce in the stratum oriens and stratum lucidum. Scale bars, $50 \mu \mathrm{m}$. $\boldsymbol{D}$, In situ hybridization of tau mRNA in the adult mouse brain. Hippocampal CA3 is shown at the same magnification of the right image in C. As in the newborn hippocampus, most signals were found in the stratum pyramidale. Scale bar, $50 \mu \mathrm{m}$.

ually. We recently developed several antibodies that can detect human and mouse tau separately at high sensitivity in brain tissues and showed precise localization of endogenous normal tau in brain tissues (Kubo et al., 2019). Using these immunological reagents, we studied how normal tau becomes axonally localized during development in vivo and what differences make human tau mislocalized in tauopathy mouse models.

The most unexpected result in this study was the dramatic suppression of tau mRNA occurring during the perinatal development. This was particularly interesting because mRNAs of other MT-related proteins (tubulins and MAPs) also showed similar reductions. The timing of mRNA suppression and the timing of axonal localization of tau occur around the same time during the first 2 weeks after birth. From these findings, we hypothesize that the production of tau protein and its axonal localization occur mostly only during the perinatal development, such that tau excessively expressed beyond this period would mislocalize and exhibit abnormal MT binding. This explains why only P301L-Tg but not P301L-KI mice exhibited the abnormal soma- todendritic localization and MT-binding of exogenous human tau.

Tau expression in the Tg mice used here is controlled by the CaMKII or mouse prion protein promoter, which resulted in the increasing expression of tau mRNA and protein beyond 2 weeks after birth. Intriguingly, this overexpression of exogenous tau did not affect the distribution and MT binding of endogenous tau, indicating that exogenous human tau proteins do not intermix with endogenous mouse tau proteins within the same neurons. This is also apparent in the STED imaging of tau in the axons. We have recently shown that endogenous mouse tau distributes sparsely on axonal MTs and speculated that axonal tau might be coassembled into MTs with tubulins rather than simply bound on them (Kar et al., 2003). This sparse distribution of endogenous tau was not affected in the Tg mice, despite that overexpressed human tau was overwhelmingly abundant in the axon. This can only be possible if the majority of abnormal exogenous tau is expressed after the completion of axonal localization of endogenous tau, as the hypothesis predicts and our data indicate. 
A

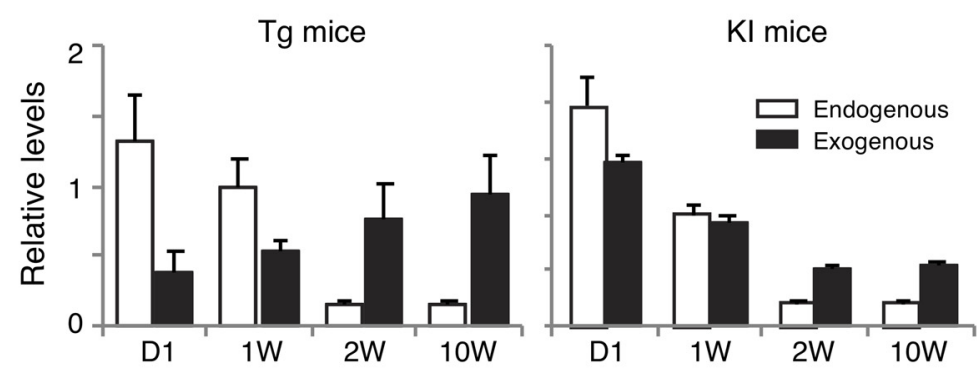

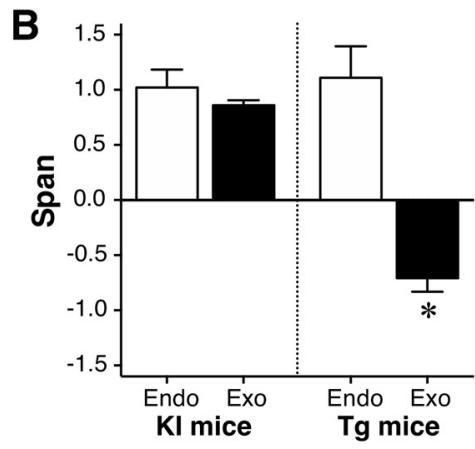

C
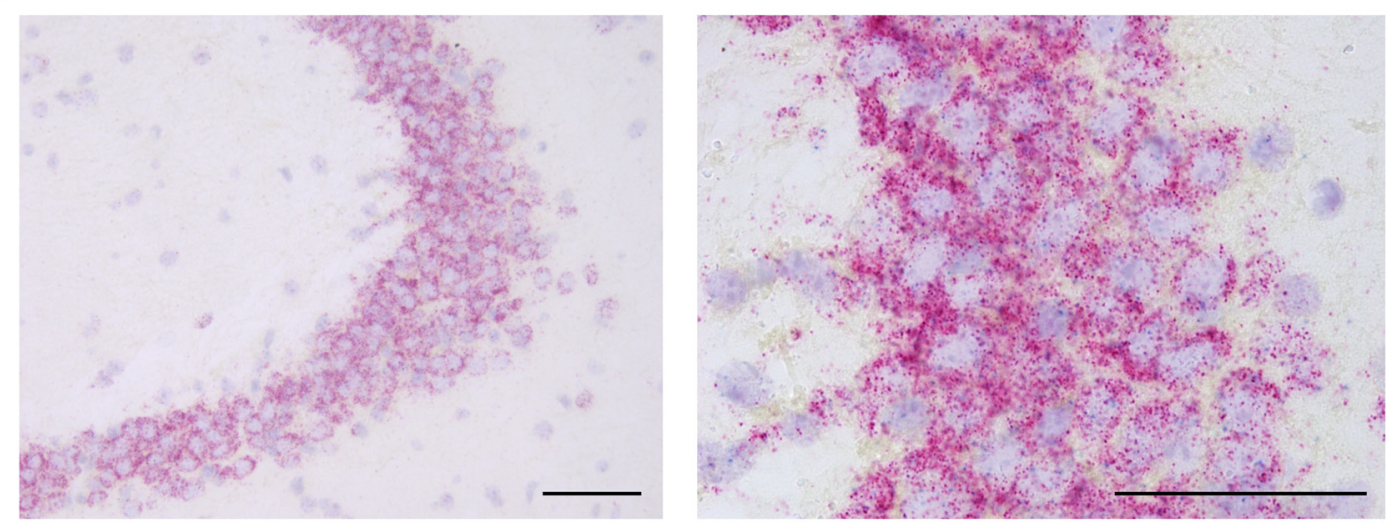

D
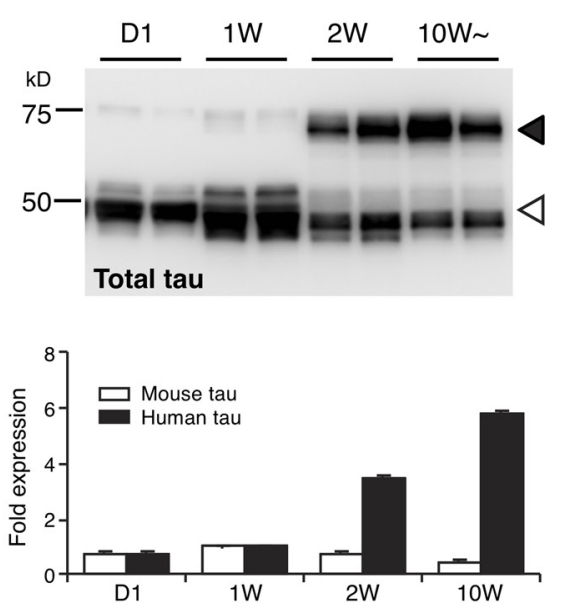

E
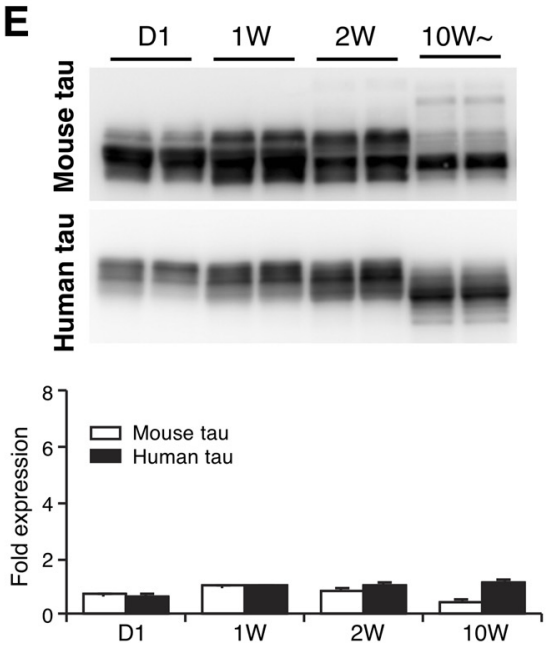

$\mathbf{F}$

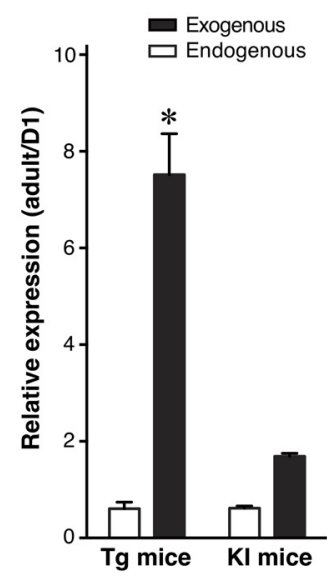

Figure 12. Abnormal expression pattern of exogenous tau in Tg mice. $\boldsymbol{A}$, Relative expression levels of endogenous mouse tau and exogenous human tau in P301L-Tg and V337M-KI mice. $\boldsymbol{B}$, Data in $\boldsymbol{A}$ were fitted with single exponential curves to compute the spans. Average spans were then compared between endogenous and exogenous tau within each model using repeated-measures two-way ANOVA with Sidak post hoc test. The difference was significant in the $\operatorname{Tg}$ mice $\left(t_{(6)}=6.13,{ }^{*} p=0.0017\right)$, but not in the KI mice $\left(t_{(6)}=0.54, p=0.8447\right) . C$, In situ hybridization of mouse and human tau mRNA in adult-transgenic mice. Brain sections from adult-transgenic mice (PS19) were subjected to in situ hybridization for endogenous tau mRNA (blue dots) and exogenous human tau mRNA (red dots) using the ViewRNA method. It should be noted that the signals of mouse mRNA were difficult to detect due to the dense signals of the human mRNA. Low-magnification (left) and high-magnification (right) views of hippocampal CA3 regions showed that most of human tau mRNA signals were still found in the perikarya of pyramidal cells. Scale bar, $50 \mu \mathrm{m}$. D, E, Protein levels of endogenous mouse tau and exogenous human tau in P301L-Tg (D) and heterozygous KI (E) mice from quantitative Western blotting. Bottom, Relative protein levels to that at 1 week for endogenous (solid) and exogenous (open) proteins individually. $F$, Ratios of protein levels at D1 and in the adulthood (adult over D1) were analyzed for Tg and KI mice. The ratio for exogenous tau in Tg mice was significantly greater than that for endogenous tau, and also significantly larger than those for endogenous tau and exogenous tau in KI mice $\left(F_{(1,14)}=58.81,{ }^{*} p<0.0001\right.$ using repeated-measures two-way ANOVA with Tukey tests), indicating that exogenous human tau is aberrantly expressed in adult Tg mice but not in KI mice.

Previous studies have shown that a small portion of tau mRNA is distributed to the axon in cultured neurons and indicated that tau may be locally translated and therefore localized in the axon (Litman et al., 1993). However, our in situ hybridization data showed that most of tau transcripts are retained in the somata in vivo. Also, the data from the KI mice suggest that the native $3^{\prime}$-untranslated region (UTR) is not neces- sary for the expression and localization of tau because the genetic construct used for the tau-KI mice was the human tau CDNA with the $3^{\prime}$-UTR of the bovine $\beta$-globin (Sadot et al., 1994). Therefore, the precise temporal regulation of expression, but not the local translation, is the key determinant of the axonal localization of tau in the physiological condition. 

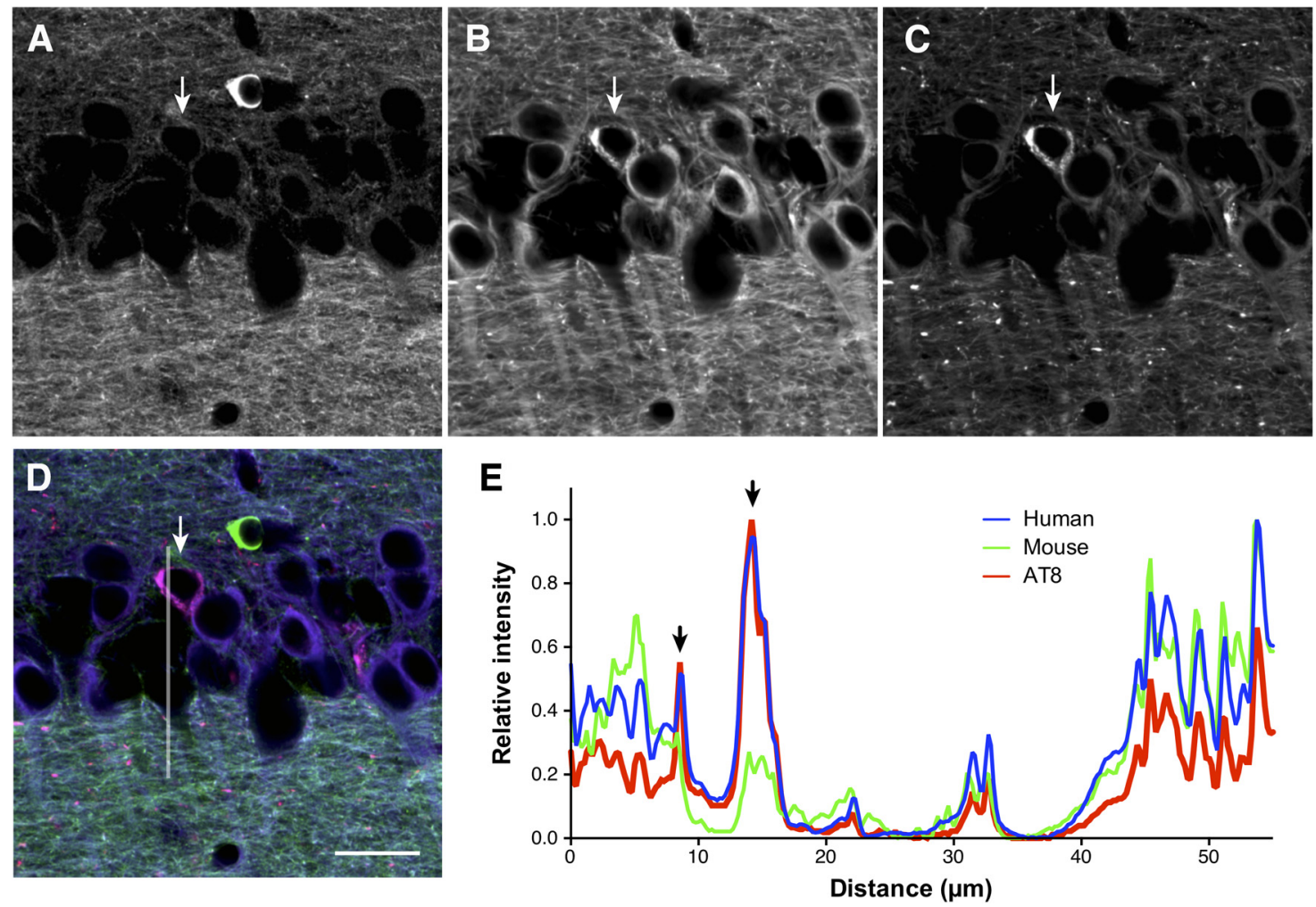

Figure 13. Tau inclusions with exogenous human tau lacking endogenous mouse tau in tau-Tg mice. $\boldsymbol{A}-\boldsymbol{D}$, Brain sections of 10-month-old PS19 mice were labeled for endogenous mouse tau (anti-rodent tauN; green; $\boldsymbol{A}$ ), exogenous human tau (RTM49; blue; $\boldsymbol{B}$ ), and AT8 (red; $\boldsymbol{C}$ ). The hippocampal CA1 is shown. A merged view is shown in $\boldsymbol{D}$. AT8-positive tau inclusions were colabeled with the antibody for human tau (arrow) but not for endogenous mouse tau. The line was used for the analysis in $\boldsymbol{E}$. Scale bar, $20 \mu \mathrm{m}$. $\boldsymbol{E}$, Line scan analysis of mouse and human tau and AT8 immunoreactivity in $\boldsymbol{D}$. The arrow indicates the position of cytoplasmic inclusions positive with AT8.
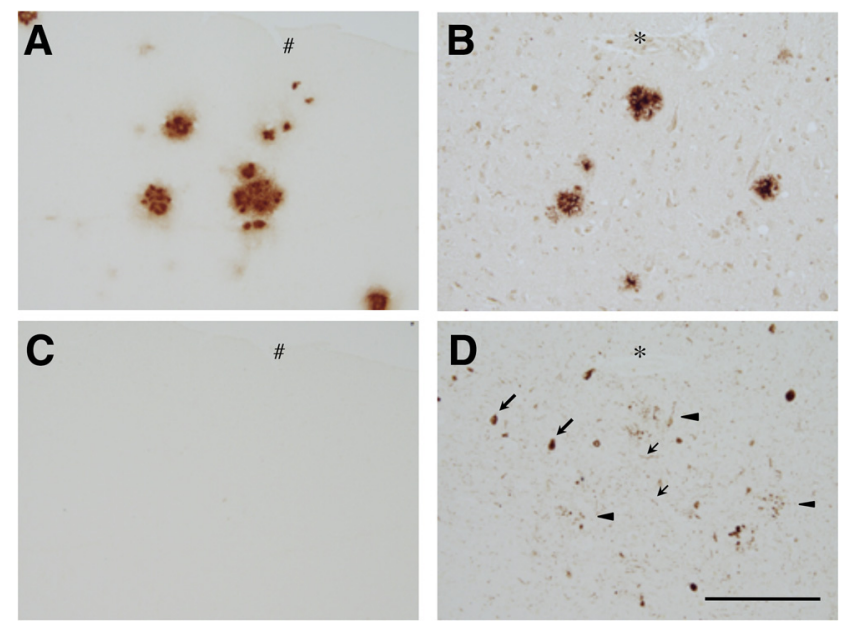

Figure 14. Lack of tau pathology in aged P301L-KI mice. Homozygous P301L-KI mice were crossbred with APP-Tg mice (Tg-2576) and the amyloid and tau pathologies were examined by immunostaining with anti-amyloid $\beta(\boldsymbol{A}, \boldsymbol{B})$ and AT8 $(\boldsymbol{C}, \boldsymbol{D})$ antibodies at 18 months of age. Sections from $A D$ brains were used for positive controls $(\boldsymbol{B}, \boldsymbol{D})$. The abundant senile plaques were detected in both aged crossbred mice and $\mathrm{AD}$ brains $(\boldsymbol{A}, \boldsymbol{B})$. Tau-related pathologies, such as NFts (large arrows), neuropil threads (small arrows), and dystrophic neurites around senile plaque (arrowheads), were found in an adjacent section of the human brain $(\boldsymbol{D})$, but none of them was detected in the mouse brain (C). Asterisks and hashmarks indicate the same blood vessels appeared on the adjacent brain sections. Scale bar, $100 \mu \mathrm{m}$.

A number of strains of tau-Tg mice that develop tau pathologies have been reported to date (Noble et al., 2010). Particularly, crossbreeding with an amyloid-producing mouse line or the injection of amyloid $\beta$ peptide into the brain can cause tau pathol- ogy even in Tg mice that express human tau at substantially lower levels (Götz et al., 2001; Lewis et al., 2001; Umeda et al., 2014). In contrast to these $\mathrm{Tg}$ mice, we were unable to find any tau pathologies in the P301L-KI mice, even when they were crossbred with the APP-Tg mice. Similar results have been reported using KI mice having mutated mouse tau genes (Gilley et al., 2016). Therefore, ectopic promoter-driven expression of exogenous tau itself is necessary in developing tau pathology in rodent brains. Furthermore, the lack of tau pathology in the tau-KI mice suggests that the abnormal distribution of tau in the somatodendritic compartment is a key step for the development of other tau pathologies. Consistently, we found that only mislocalized human tau in PS19 mice, but not axonal mouse tau, contributed to the formation of tau inclusions. Together, our study suggests that the dysfunction of the strict transcriptional regulation due to aging or by other factors could be a potential cause of tau mislocalization and abnormal MT binding, which lead to other pathological changes in tauopathies including AD.

Although most tau inclusions are formed in cell bodies and dendrites (Attems, 2013), axonal tau inclusions have also been reported as a subset of dystrophic neurites surrounding senile plaque in AD and FTDP-17 (Tourtellotte and Van Hoesen, 1991; Kneynsberg et al., 2017; Ono et al., 2017). Furthermore, a study by Christensen et al. (2019) recently reported that, in human patient postmortem tissues, phosphorylated and/or misfolded tau can be detected in the axons of hippocampal neurons in the absence of these pathological tau species in the somata, indicating that tau pathologies can occur without its mislocalization. These findings raise the questions of where tau pathology first emerges and whether tau mislocalizationin somatodendrites precedes 
such visible tau abnormalities. Most of past research has focused on phosphorylated, misfolded, and/or aggregated tau, and it has not been extensively studied how tau changes its overall subcellular localization in animal models and postmortem tissues. This is presumably due to the lack of antibodies, which can detect normal physiological tau without extensive phosphorylation and misfolding. The antibodies used in this study can label tau regardless of phosphorylation in human and animal tissues. Therefore, it would be an important next question to address where and in what order these pathological changes of tau (mislocalization, phosphorylation, misfolding, and aggregation) occur in $\mathrm{AD}$ patients.

For somatodendritic tau inclusions in animal models, however, our results from $\mathrm{Tg}$ and KI mice with the same FTDP mutation indicate that mislocalization is necessary. It may not be sufficient. Even though human tau mislocalizes throughout the life span of Tg mice, tau pathology appears only when the animals age. Also, tau protein is stable and does not aggregate itself in vitro without inducers (Goedert et al., 1996). Other changes and factors associated with aging may also be necessary to start tau aggregation. Hyperphosphorylation has been implicated as a major one of such changes among many others including ubiquitination, truncation, acetylation, glycosylation, etc. (Abraha et al., 2000; Zilka et al., 2006; Cohen et al., 2011; Martin et al., 2011). However, hyperphosphorylation itself does not seem to be sufficient, as evidenced by the lack of tau inclusions in hypothermia mice, in which hyperphosphorylation of endogenous tau is induced (Planel et al., 2007). A recent study (Gilley et al., 2016) investigated KI mice with hyperphosphorylation and hypophosphorylation mimetic tau mutants and reported their mislocalization only in aged animals. Although these findings indicate that hyperphosphorylation or hypophosphorylation could potentially cause mislocalization of tau, it is not sufficient to cause tau inclusions (Gilley et al., 2016). In the light of our findings that dysregulation of tau expression may be a key, further studies are necessary to determine critical factors inducing somatodendritic tau inclusions.

Our hypothesis based on the great reduction of tau transcripts after early development requires that the tau proteins generated during the perinatal period is mostly maintained for a long time without significant turnover. This would make tau one of the most extremely long-lived proteins in the brain (Toyama and Hetzer, 2013; Toyama et al., 2013). These proteins, such as collagens and histones, are produced mostly during early development and maintained for a long time, sometimes for the lifetime, with very limited turnover. Because ectopically expressed tau behaves abnormally, making tau early and long-living may be critical for maintaining the physiological function of tau in long axons, particularly unmyelinated axons, where we observed robust tau labeling (Kubo et al., 2019). However, the evidence for tau to be one of these long-lived proteins is currently limited to studies using old radiolabeling methods (Tashiro et al., 1996) and those using stable-isotope labeling methods but focusing on either extracellular tau or the whole proteome (Sato et al., 2018). Studies targeting intracellular tau, which should be further separated into MT-bound and MT-unbound tau as well as glial and neuronal tau, would be necessary to investigate the turnover rate of tau in neurons.

\section{References}

Abraha A, Ghoshal N, Gamblin TC, Cryns V, Berry RW, Kuret J, Binder LI (2000) C-terminal inhibition of tau assembly in vitro and in Alzheimer's disease. J Cell Sci 113:3737-3745.
Amaral DG, Scharfman HE, Lavenex P (2007) The dentate gyrus: fundamental neuroanatomical organization (dentate gyrus for dummies). Prog Brain Res 163:3-22.

Attems J, Kurt AJ (2013) Neuropathology. In: Oxford textbook of old age psychiatry, Ed 2 (Thomas TDaA, ed), pp 87-105. Oxford: OUP.

Binder LI, Frankfurter A, Rebhun LI (1985) The distribution of tau in the mammalian central nervous system. J Cell Biol 101:1371-1378.

Braak H, Braak E. (1994) Morphological criteria for the recognition of Alzheimer's disease and the distribution pattern of cortical changes related to this disorder. Neurobiol Aging 15:355-356; discussion 379-380.

Christensen KR, Beach TG, Serrano GE, Kanaan NM (2019) Pathogenic tau modifications occur in axons before the somatodendritic compartment in mossy fiber and Schaffer collateral pathways. Acta Neuropathol Commun 7:29.

Cohen TJ, Guo JL, Hurtado DE, Kwong LK, Mills IP, Trojanowski JQ, Lee VM (2011) The acetylation of tau inhibits its function and promotes pathological tau aggregation. Nat Commun 2:252.

Dawson HN, Ferreira A, Eyster MV, Ghoshal N, Binder LI, Vitek MP (2001) Inhibition of neuronal maturation in primary hippocampal neurons from tau deficient mice. J Cell Sci 114:1179-1187.

Delacourte A, David JP, Sergeant N, Buée L, Wattez A, Vermersch P, Ghozali F, Fallet-Bianco C, Pasquier F, Lebert F, Petit H, Di Menza C (1999) The biochemical pathway of neurofibrillary degeneration in aging and Alzheimer's disease. Neurology 52:1158-1165.

Deshpande A, Win KM, Busciglio J (2008) Tau isoform expression and regulation in human cortical neurons. FASEB J 22:2357-2367.

Faul F, Erdfelder E, Lang AG, Buchner A (2007) G*Power 3: a flexible statistical power analysis program for the social, behavioral, and biomedical sciences. Behav Res Methods 39:175-191.

Faul F, Erdfelder E, Buchner A, Lang AG (2009) Statistical power analyses using $G^{\star}$ Power 3.1: tests for correlation and regression analyses. Behav Res Methods 41:1149-1160.

Frandemiche ML, De Seranno S, Rush T, Borel E, Elie A, Arnal I, Lanté F, Buisson A (2014) Activity-dependent tau protein translocation to excitatory synapse is disrupted by exposure to amyloid-beta oligomers. J Neurosci 34:6084-6097.

Ghetti B, Oblak AL, Boeve BF, Johnson KA, Dickerson BC, Goedert M (2015) Invited review: frontotemporal dementia caused by microtubuleassociated protein tau gene (MAPT) mutations: a chameleon for neuropathology and neuroimaging. Neuropathol Appl Neurobiol 41:24-46.

Gilley J, Ando K, Seereeram A, Rodríguez-Martín T, Pooler AM, Sturdee L, Anderton BH, Brion JP, Hanger DP, Coleman MP (2016) Mislocalization of neuronal tau in the absence of tangle pathology in phosphomutant tau knockin mice. Neurobiol Aging 39:1-18.

Goedert M, Jakes R, Spillantini MG, Hasegawa M, Smith MJ, Crowther RA (1996) Assembly of microtubule-associated protein tau into alzheimerlike filaments induced by sulphated glycosaminoglycans. Nature 383: $550-553$.

Gómez-Isla T, Hollister R, West H, Mui S, Growdon JH, Petersen RC, Parisi JE, Hyman BT (1997) Neuronal loss correlates with but exceeds neurofibrillary tangles in Alzheimer's disease. Ann Neurol 41:17-24.

Götz J, Chen F, van Dorpe J, Nitsch RM (2001) Formation of neurofibrillary tangles in P3011 tau-transgenic mice induced by abeta 42 fibrils. Science 293:1491-1495.

Harada A, Oguchi K, Okabe S, Kuno J, Terada S, Ohshima T, Sato-Yoshitake R, Takei Y, Noda T, Hirokawa N (1994) Altered microtubule organization in small-calibre axons of mice lacking tau protein. Nature 369:488491.

Hsiao K, Chapman P, Nilsen S, Eckman C, Harigaya Y, Younkin S, Yang F, Cole G (1996) Correlative memory deficits, Abeta elevation, and amyloid plaques in-transgenic mice. Science 274:99-102.

Iqbal K, Liu F, Gong CX (2016) Tau and neurodegenerative disease: the story so far. Nat Rev Neurol 12:15-27.

Johnson GV, Jenkins SM (1999) Tau protein in normal and Alzheimer's disease brain. J Alzheimers Dis 1:307-328.

Kaech S, Banker G (2006) Culturing hippocampal neurons. Nat Protoc 1:2406-2415.

Kar S, Fan J, Smith MJ, Goedert M, Amos LA (2003) Repeat motifs of tau bind to the insides of microtubules in the absence of Taxol. EMBO J 22:70-77.

Kimura T, Yamashita S, Fukuda T, Park JM, Murayama M, Mizoroki T, Yoshiike Y, Sahara N, Takashima A (2007) Hyperphosphorylated tau in 
parahippocampal cortex impairs place learning in aged mice expressing wild-type human tau. EMBO J 26:5143-5152.

Kimura T, Fukuda T, Sahara N, Yamashita S, Murayama M, Mizoroki T, Yoshiike Y, Lee B, Sotiropoulos I, Maeda S, Takashima A (2010) Aggregation of detergent-insoluble tau is involved in neuronal loss but not in synaptic loss. J Biol Chem 285:38692-38699.

Kneynsberg A, Combs B, Christensen K, Morfini G, Kanaan NM (2017) Axonal degeneration in tauopathies: disease relevance and underlying mechanisms. Front Neurosci 11:572.

Kowall NW, Kosik KS (1987) Axonal disruption and aberrant localization of tau protein characterize the neuropil pathology of Alzheimer's disease. Ann Neurol 22:639-643.

Kubo A, Misonou H, Matsuyama M, Nomori A, Wada-Kakuda S, Takashima A, Kawata M, Murayama S, Ihara Y, Miyasaka T (2019) Distribution of endogenous normal tau in the mouse brain. J Comp Neurol 527:985-998.

Kuchibhotla KV, Wegmann S, Kopeikina KJ, Hawkes J, Rudinskiy N, Andermann ML, Spires-Jones TL, Bacskai BJ, Hyman BT (2014) Neurofibrillary tangle-bearing neurons are functionally integrated in cortical circuits in vivo. Proc Natl Acad Sci U S A 111:510-514.

Lewis J, Dickson DW, Lin WL, Chisholm L, Corral A, Jones G, Yen SH, Sahara N, Skipper L, Yager D, Eckman C, Hardy J, Hutton M, McGowan E (2001) Enhanced neurofibrillary degeneration in-transgenic mice expressing mutant tau and APP. Science 293:1487-1491.

Litman P, Barg J, Rindzoonski L, Ginzburg I (1993) Subcellular localization of tau mRNA in differentiating neuronal cell culture: implications for neuronal polarity. Neuron 10:627-638.

Martin L, Page G, Terro F (2011) Tau phosphorylation and neuronal apoptosis induced by the blockade of PP2A preferentially involve GSK3beta. Neurochem Int 59:235-250.

Matsumura N, Yamazaki T, Ihara Y (1999) Stable expression in chinese hamster ovary cells of mutated tau genes causing frontotemporal dementia and parkinsonism linked to chromosome 17 (FTDP-17). Am J Pathol 154:1649-1656.

Misonou H, Trimmer JS (2005) A primary culture system for biochemical analyses of neuronal proteins. J Neurosci Methods 144:165-173.

Miyasaka T, Ding Z, Gengyo-Ando K, Oue M, Yamaguchi H, Mitani S, Ihara Y (2005a) Progressive neurodegeneration in C. elegans model of tauopathy. Neurobiol Dis 20:372-383.

Miyasaka T, Watanabe A, Saito Y, Murayama S, Mann DM, Yamazaki M, Ravid R, Morishima-Kawashima M, Nagashima K, Ihara Y (2005b) Visualization of newly deposited tau in neurofibrillary tangles and neuropil threads. J Neuropathol Exp Neurol 64:665-674.

Miyasaka T, Sato S, Tatebayashi Y, Takashima A (2010) Microtubule destruction induces tau liberation and its subsequent phosphorylation. FEBS Lett 584:3227-3232.

Noble W, Hanger DP, Gallo JM (2010) Transgenic mouse models of tauopathy in drug discovery. CNS Neurol Disord Drug Targets 9:403-428.

Ono M, Sahara N, Kumata K, Ji B, Ni R, Koga S, Dickson DW, Trojanowski JQ, Lee VM, Yoshida M, Hozumi I, Yoshiyama Y, van Swieten JC, Nordberg A, Suhara T, Zhang MR, Higuchi M (2017) Distinct binding of PET ligands PBB3 and AV-1451 to tau fibril strains in neurodegenerative tauopathies. Brain 140:764-780.

Planel E, Yasutake K, Fujita SC, Ishiguro K (2001) Inhibition of protein phosphatase $2 \mathrm{~A}$ overrides tau protein kinase I/glycogen synthase kinase 3 beta and cyclin-dependent kinase 5 inhibition and results in tau hyperphosphorylation in the hippocampus of starved mouse. J Biol Chem 276:34298-34306.

Planel E, Richter KE, Nolan CE, Finley JE, Liu L, Wen Y, Krishnamurthy P,
Herman M, Wang L, Schachter JB, Nelson RB, Lau LF, Duff KE (2007) Anesthesia leads to tau hyperphosphorylation through inhibition of phosphatase activity by hypothermia. J Neurosci 27:3090-3097.

Sadot E, Marx R, Barg J, Behar L, Ginzburg I (1994) Complete sequence of $3^{\prime}$ - untranslated region of tau from rat central nervous system: implications for mRNA heterogeneity. J Mol Biol 241:325-331.

Santacruz K, Lewis J, Spires T, Paulson J, Kotilinek L, Ingelsson M, Guimaraes A, DeTure M, Ramsden M, McGowan E, Forster C, Yue M, Orne J, Janus C, Mariash A, Kuskowski M, Hyman B, Hutton M, Ashe KH (2005) Tau suppression in a neurodegenerative mouse model improves memory function. Science 309:476-481.

Sato C, Barthélemy NR, Mawuenyega KG, Patterson BW, Gordon BA, JockelBalsarotti J, Sullivan M, Crisp MJ, Kasten T, Kirmess KM, Kanaan NM, Yarasheski KE, Baker-Nigh A, Benzinger TLS, Miller TM, Karch CM, Bateman RJ (2018) Tau kinetics in neurons and the human central nervous system. Neuron 98:861-864.

Scholz T, Mandelkow E (2014) Transport and diffusion of tau protein in neurons. Cell Mol Life Sci 71:3139-3150.

Tashiro T, Sun X, Tsuda M, Komiya Y (1996) Differential axonal transport of soluble and insoluble tau in the rat sciatic nerve. J Neurochem 67:1566-1574.

Tourtellotte WG, Van Hoesen GW (1991) The axonal origin of a subpopulation of dystrophic neurites in Alzheimer's disease. Neurosci Lett 129:11-16.

Toyama BH, Hetzer MW (2013) Protein homeostasis: live long, won't prosper. Nat Rev Mol Cell Biol 14:55-61.

Toyama BH, Savas JN, Park SK, Harris MS, Ingolia NT, Yates JR 3rd, Hetzer MW (2013) Identification of long-lived proteins reveals exceptional stability of essential cellular structures. Cell 154:971-982.

Trojanowski JQ, Schuck T, Schmidt ML, Lee VM (1989) Distribution of tau proteins in the normal human central and peripheral nervous system. J Histochem Cytochem 37:209-215.

Umeda T, Yamashita T, Kimura T, Ohnishi K, Takuma H, Ozeki T, Takashima A, Tomiyama T, Mori H (2013) Neurodegenerative disorder FTDP-17-related tau intron $10+16 \mathrm{C}->$ T mutation increases tau exon 10 splicing and causes tauopathy in-transgenic mice. Am J Pathol 183:211-225

Umeda T, Maekawa S, Kimura T, Takashima A, Tomiyama T, Mori H (2014) Neurofibrillary tangle formation by introducing wild-type human tau into APP-transgenic mice. Acta Neuropathol 127:685-698.

Viereck C, Tucker RP, Binder LI, Matus A (1988) Phylogenetic conservation of brain microtubule-associated proteins MAP2 and tau. Neuroscience 26:893-904.

Yoshiyama Y, Higuchi M, Zhang B, Huang SM, Iwata N, Saido TC, Maeda J, Suhara T, Trojanowski JQ, Lee VM (2007) Synapse loss and microglial activation precede tangles in a P301S tauopathy mouse model. Neuron 53:337-351.

Zempel H, Mandelkow E (2014) Lost after translation: missorting of tau protein and consequences for Alzheimer disease. Trends Neurosci 37:721-732.

Zempel H, Thies E, Mandelkow E, Mandelkow EM (2010) Abeta oligomers cause localized $\mathrm{Ca}(2+)$ elevation, missorting of endogenous tau into dendrites, Tau phosphorylation, and destruction of microtubules and spines. J Neurosci 30:11938-11950.

Zilka N, Filipcik P, Koson P, Fialova L, Skrabana R, Zilkova M, Rolkova G, Kontsekova E, Novak M (2006) Truncated tau from sporadic Alzheimer's disease suffices to drive neurofibrillary degeneration in vivo. FEBS Lett 580:3582-3588. 